\title{
Periodic solutions for quaternion-valued fuzzy cellular neural networks with time-varying delays
}

\author{
Yongkun $\mathrm{Li}^{1 *}$ (D), Jiali Qin' ${ }^{1}$ and Bing $\mathrm{Li}^{2}$
}

"Correspondence: yklie@ynu.edu.cn 'Department of Mathematics, Yunnan University, Yunnan, China Full list of author information is available at the end of the article

\begin{abstract}
In this paper, quaternion-valued fuzzy cellular neural networks (QVFCNNs) with time-varying delays are considered. First, we decompose QVFCNNs into their equivalent real-valued systems according to Hamilton's multiplication rules. Then, we establish the existence and global exponential stability of periodic solutions of QVFCNNs by using the Schauder fixed point theorem and by constructing an appropriate Lyapunov function. Our results are completely new and supplementary to the known results. Finally, we give a numerical example to illustrate the effectiveness of our results.
\end{abstract}

Keywords: Quaternion-valued fuzzy cellular neural networks; Schauder fixed point theorem; Periodic solution; Global exponential stability; Time-varying delays

\section{Introduction}

Quaternion was invented by the Irish mathematician W. R. Hamilton in 1843 [1]. The skew field of quaternion is denoted by

$$
\mathbb{H}:=\left\{q=q_{0}+i q_{1}+j q_{2}+k q_{3}\right\}
$$

where $q_{0}, q_{1}, q_{2}, q_{3}$ are real numbers and the elements $i, j$, and $k$ obey Hamilton's multiplication rules:

$$
i j=-j i=k, \quad j k=-k j=i, \quad k i=-i k=j, \quad i^{2}=j^{2}=k^{2}=i j k=-1 .
$$

Quaternion multiplication does not conform to the law of commutation, so the study on quaternion is much more difficult than that on plurality. Fortunately, over the past 20 years, especially in algebra area, quaternion has been a hot topic for the effective applications in the real world. Also, a new class of differential equations, named quaternion differential equations, has been already applied successfully to the fields such as quantum mechanics [2, 3], robotic manipulation [4], fluid mechanics [5], differential geometry [6], communications problems, signal processing [7-9], and neural networks [10-13]. In particular, in recent years, the applications of quaternion-valued neural networks (QVNNs),

(c) The Author(s) 2019. This article is distributed under the terms of the Creative Commons Attribution 4.0 International License (http://creativecommons.org/licenses/by/4.0/), which permits unrestricted use, distribution, and reproduction in any medium, provided you give appropriate credit to the original author(s) and the source, provide a link to the Creative Commons license, and indicate if changes were made. 
which are described by quaternion-valued differential equations, have been widely investigated. One practical application by QVNNs is the 3D geometrical affine transformation, especially spatial rotation, which can be represented based on QVNNs efficiently and compactly $[14,15]$. Other practical applications of QVNNs are image impression, color night vision [16], etc.

On the one hand, it is well known that neural networks have been extensively studied for their wide application in image processing, pattern recognition, optimization solvers, artificial intelligence, fixed point calculations, and other engineering fields [17-20]. These applications rely heavily on their dynamics. Many scholars tried to shed some light on the information about the dynamics of QVNNs. For example, authors of [21] studied the global $\mu$-stability criteria for quaternion-valued neural networks with unbounded time-varying delays; from the view of matrix measure, based on Halanay inequality instead of Lyapunov function, authors of [22] derived some sufficient conditions to guarantee the global exponential stability for QVNNs; authors of [23] investigated the periodicity of QVNNs by a continuation theorem of coincidence degree theory; authors of [24] studied the existence of pseudo almost periodic solutions of QVNNs. However, as we all know, up to now, there have been few results about the dynamics of quaternion-valued neural networks.

On the other hand, fuzzy cellular neural networks (FCNNs) proposed by Yang and Yang in 1996 [25] have been successfully applied in many fields such as physics, chemistry, biology, economics, sociology, medicine, meteorology, and so on [26]. In recent years, many researchers have done a lot of research on the solutions of general fuzzy differential equations and the dynamics of FCNNs (see [27-47] and the references therein). As is known, periodic oscillations and almost periodic oscillations are important dynamical behaviors of non-autonomous neural networks. However, as far as we know, no scholars have studied the periodic solutions of quaternion-valued fuzzy cellular neural networks (QVFCNNs).

Considering the discussion above, in this paper, we are committed to studying the following QVFCNN with time-varying delays:

$$
\begin{aligned}
\dot{x}_{p}(t)= & -a_{p}(t) x_{p}(t)+\sum_{q=1}^{n} b_{p q}(t) f_{q}\left(x_{q}\left(t-\tau_{p q}(t)\right)\right)+\sum_{q=1}^{n} d_{p q}(t) \mu_{q}(t) \\
& +\bigwedge_{q=1}^{n} \alpha_{p q}(t) \int_{t-\eta_{p q}(t)}^{t} g_{q}(s, x) d s+\bigvee_{q=1}^{n} \beta_{p q}(t) \int_{t-\xi p q}^{t} g_{q}(s, x) d s \\
& +\bigwedge_{q=1}^{n} T_{p q}(t) \mu_{q}(t)+\bigvee_{q=1}^{n} S_{p q}(t) \mu_{q}(t)+I_{p}(t),
\end{aligned}
$$

where $p=1,2, \ldots, n, n$ is the number of neurons in layers; $x_{p}(t) \in \mathbb{H}$ is the state of the $p$ th neuron at time $t$, and $\mu_{q}(t) \in \mathbb{H}$ is the deviations of the $q$ th neuron at time $t ; a_{p}(t)>0$ represents the rate at which the $p$ th neuron will reset its potential to the resting state in isolation when it is disconnected from the network and the external inputs at time $t$, $\alpha_{p q}(t) \in \mathbb{H}, \beta_{p q}(t) \in \mathbb{H}, T_{p q}(t) \in \mathbb{H}$, and $S_{p q}(t) \in \mathbb{H}$ are the elements of fuzzy feedback MIN template, fuzzy feedback MAX template, fuzzy feed forward MIN template, and fuzzy feed forward MAX template, respectively; $b_{p q}(t) \in \mathbb{H}$ and $d_{p q}(t) \in \mathbb{H}$ are the elements of feedback template and feed forward template, $\bigwedge, \bigvee$ denote the fuzzy AND and fuzzy OR operations, respectively; $f_{q}(\cdot)$ and $g_{q}(\cdot): \mathbb{H} \rightarrow \mathbb{H}$ are the activation functions; $\tau_{p q}(t) \geq 0$, 
$\eta_{p q}(t) \geq 0$, and $\xi_{p q}(t) \geq 0$ correspond to transmission delays at time $t ; I_{p}(t) \in \mathbb{H}$ denotes the input of the $p$ th neuron at time $t$.

Our main purpose of this paper is by using the Schauder fixed point theorem and constructing an appropriate Lyapunov function to study the existence, the uniqueness, and the global exponential stability of periodic solutions of (1). Our results are completely new and when (1) degenerates into real-valued system, our results remain new and supplementary to the known results obtained in [23, 32, 45-47], and our method used in this paper is different from those used in [23, 32, 45-47].

The rest of this paper is organized as follows. In Sect. 2, we introduce some symbols and definitions and present some preliminary results needed later. In Sect. 3, we establish some sufficient conditions for the existence and global exponential stability of periodic solutions of (1). In Sect. 4, an example is given to illustrate the effectiveness of the results.

\section{Preliminaries}

In this section, we introduce some definitions, notions, lemmas and decompose (1) into an equivalent real-valued system.

Definition 1 Let $h=h^{R}+i h^{I}+j h^{J}+h^{K}: \mathbb{R} \rightarrow \mathbb{H}$, where $h^{l}: \mathbb{R} \rightarrow \mathbb{H}, l \in\{R, I, J, K\}:=\Upsilon . h$ is said to be $\omega$-anti-periodic if for every $l \in \Upsilon, h^{l}: \mathbb{R} \rightarrow \mathbb{R}$ is $\omega$-anti-periodic on $\mathbb{R}$.

Lemma 1 ([48] Schauder fixed point theorem) Let $E$ be a normed space, and let $C$ be a convex, bounded, and closed subset of $E$. If $T: C \rightarrow C$ is continuous with $\overline{T(C)}$ compact, then $T$ has at least one fixed point.

Throughout this paper, we assume the following.

$\left(H_{1}\right)$ Let $x_{p}=x_{p}^{R}+i x_{p}^{I}+j x_{p}^{J}+k x_{p}^{K}, x_{p}^{R}, x_{p}^{I}, x_{p}^{J}, x_{p}^{K}: \mathbb{R} \rightarrow \mathbb{R}$ and assume $f_{p}\left(x_{p}\right)$ and $g_{p}\left(x_{p}\right)$ can be expressed as

$$
\begin{aligned}
f_{p}\left(x_{p}\right)= & f_{p}^{R}\left(x_{p}^{R}, x_{p}^{I}, x_{p}^{J}, x_{p}^{K}\right)+i f_{p}^{I}\left(x_{p}^{R}, x_{p}^{I}, x_{p}^{J}, x_{p}^{K}\right) \\
& +j f_{p}^{J}\left(x_{p}^{R}, x_{p}^{I}, x_{p}^{J}, x_{p}^{K}\right)+k f_{p}^{K}\left(x_{p}^{R}, x_{p}^{I}, x_{p}^{J}, x_{p}^{K}\right), \\
g_{p}\left(x_{p}\right)= & g_{p}^{R}\left(x_{p}^{R}, x_{p}^{I}, x_{p}^{J}, x_{p}^{K}\right)+i g_{p}^{I}\left(x_{p}^{R}, x_{p}^{I}, x_{p}^{J}, x_{p}^{K}\right) \\
& +j g_{p}^{J}\left(x_{p}^{R}, x_{p}^{I}, x_{p}^{J}, x_{p}^{K}\right)+k g_{p}^{K}\left(x_{p}^{R}, x_{p}^{I}, x_{p}^{J}, x_{p}^{K}\right),
\end{aligned}
$$

where $f_{p}^{l}, g_{p}^{l}: \mathbb{R}^{4} \rightarrow \mathbb{R}, p=1,2, \ldots, n, l \in \Upsilon$.

$\left(H_{2}\right)$ Functions $a_{p}, \tau_{p q}, \eta_{p q}, \xi_{p q} \in C\left(\mathbb{R}, \mathbb{R}^{+}\right), b_{p q}, d_{p q}, \alpha_{p q}, \beta_{p q}, T_{p q}, S_{p q}, \mu_{q}, I_{p} \in C(\mathbb{R}, \mathbb{H})$ are $\omega$-periodic functions, where $x_{q}^{l} \in \mathbb{R}, p, q=1,2, \ldots, n$.

$\left(H_{3}\right)$ Functions $f_{q}^{l}, g_{q}^{l} \in C\left(\mathbb{R}^{4}, \mathbb{R}\right)$ and there exist positive constants $L_{f}, L_{g}$ such that, for all $x_{q}^{l}, y_{q}^{l} \in \mathbb{R}$

$$
\begin{aligned}
& \left|f_{q}^{l}\left(x_{q}^{R}, x_{q}^{I}, x_{q}^{J}, x_{q}^{K}\right)-f_{q}^{l}\left(y_{q}^{R}, y_{q}^{I}, y_{q}^{J}, y_{q}^{K}\right)\right| \\
& \quad \leq L_{f}\left(\left|x_{q}^{R}-y_{q}^{R}\right|+\left|x_{q}^{I}-y_{q}^{I}\right|+\left|x_{q}^{J}-y_{q}^{J}\right|+\left|x_{q}^{K}-y_{q}^{K}\right|\right), \\
& \left|g_{h}^{l}\left(x_{q}^{R}, x_{q}^{I}, x_{q}^{J}, x_{q}^{K}\right)-g_{q}^{l}\left(y_{q}^{R}, y_{q}^{I}, y_{q}^{J}, y_{q}^{K}\right)\right| \\
& \quad \leq L_{g}\left(\left|x_{q}^{R}-y_{q}^{R}\right|+\left|x_{q}^{I}-y_{q}^{I}\right|+\left|x_{q}^{J}-y_{q}^{J}\right|+\left|x_{q}^{K}-y_{q}^{K}\right|\right)
\end{aligned}
$$

and $f_{q}^{l}(0,0,0,0)=0, g_{q}^{l}(0,0,0,0)=0, q=1,2, \ldots, n, l \in \Upsilon$. 
For $p, q=1,2, \ldots, n$, assume that

$$
\begin{array}{ll}
b_{p q}=b_{p q}^{R}+i b_{p q}^{I}+j b_{p q}^{J}+k b_{p q}^{K}, & d_{p q}=d_{p q}^{R}+i d_{p q}^{I}+j d_{p q}^{J}+k d_{p q}^{K}, \\
\alpha_{p q}=\alpha_{p q}^{R}+i \alpha_{p q}^{I}+j \alpha_{p q}^{J}+k \alpha_{p q}^{K}, & \beta_{p q}=\beta_{p q}^{R}+i \beta_{p q}^{I}+j \beta_{p q}^{J}+k \beta_{p q}^{K}, \\
T_{p q}=T_{p q}^{R}+i T_{p q}^{I}+j T_{p q}^{J}+k T_{p q}^{K}, & S_{p q}=S_{p q}^{R}+i S_{p q}^{I}+j S_{p q}^{J}+k S_{p q}^{K}, \\
\mu_{q}=\mu_{q}^{R}+i \mu_{q}^{I}+j \mu_{q}^{J}+k \mu_{q}^{K}, & I_{p}=I_{p}^{R}+i I_{p}^{I}+j I_{p}^{J}+k I_{p}^{K} .
\end{array}
$$

We will adopt the following notations:

$$
\begin{aligned}
& b_{p q}=\sup _{t \in \mathbb{R}}\left\{\left|b_{p q}^{R}(t)\right|,\left|b_{p q}^{I}(t)\right|,\left|b_{p q}^{J}(t)\right|,\left|b_{p q}^{K}(t)\right|\right\}, \\
& d_{p q}=\sup _{t \in \mathbb{R}}\left\{\left|d_{p q}^{R}(t)\right|,\left|d_{p q}^{I}(t)\right|,\left|d_{p q}^{J}(t)\right|,\left|d_{p q}^{K}(t)\right|\right\}, \\
& \alpha_{p q}=\sup _{t \in \mathbb{R}}\left\{\left|\alpha_{p q}^{R}(t)\right|,\left|\alpha_{p q}^{I}(t)\right|,\left|\alpha_{p q}^{J}(t)\right|,\left|\alpha_{p q}^{K}(t)\right|\right\}, \\
& \beta_{p q}=\sup _{t \in \mathbb{R}}\left\{\left|\beta_{p q}^{R}(t)\right|,\left|\beta_{p q}^{I}(t)\right|,\left|\beta_{p q}^{J}(t)\right|,\left|\beta_{p q}^{K}(t)\right|\right\}, \\
& T_{p q}=\sup _{t \in \mathbb{R}}\left\{\left|T_{p q}^{R}(t)\right|,\left|T_{p q}^{I}(t)\right|,\left|T_{p q}^{J}(t)\right|,\left|T_{p q}^{K}(t)\right|\right\}, \\
& S_{p q}=\sup _{t \in \mathbb{R}}\left\{\left|S_{p q}^{R}(t)\right|,\left|S_{p q}^{I}(t)\right|,\left|S_{p q}^{J}(t)\right|,\left|S_{p q}^{K}(t)\right|\right\}, \\
& \mu_{q}=\sup _{t \in \mathbb{R}}\left\{\left|\mu_{q}^{R}(t)\right|,\left|\mu_{q}^{I}(t)\right|,\left|\mu_{q}^{J}(t)\right|,\left|\mu_{q}^{K}(t)\right|\right\}, \\
& I_{p}=\sup _{t \in \mathbb{R}}\left\{\left|I_{p}^{R}(t)\right|,\left|I_{p}^{I}(t)\right|,\left|I_{p}^{J}(t)\right|,\left|I_{p}^{K}(t)\right|\right\}, \\
& \bar{a}_{p}=\frac{1}{\omega} \int_{0}^{\omega} a_{p}(t) d t, \quad a_{p}^{-}=\min _{t \in \mathbb{R}}\left\{a_{p}(t)\right\}, \\
& \tau_{p q}=\sup _{t \in \mathbb{R}}\left\{\tau_{p q}(t)\right\}, \quad \eta_{p q}=\sup _{t \in \mathbb{R}}\left\{\eta_{p q}(t)\right\}, \\
& \xi_{p q}=\sup _{t \in \mathbb{R}}\left\{\xi_{p q}(t)\right\}, \quad \dot{\tau}_{p q}=\sup _{t \in \mathbb{R}}\left\{\dot{\tau}_{p q}(t)\right\}, \\
& \rho^{+}=\max _{\substack{1 \leq q \leq n \\
l \in \Upsilon}}\left\{\rho_{p_{l}}\right\}, \quad \rho^{-}=\min _{\substack{1 \leq q \leq n \\
l \in \Upsilon}}\left\{\rho_{p_{l}}\right\} .
\end{aligned}
$$

The initial value of system (1) is given by

$$
\begin{aligned}
& x_{p}(t)=\varphi_{p}(t) \in \mathbb{H}, \quad t \in\left[t_{0}-\theta, t_{0}\right], \quad \theta=\max _{1 \leq p, q \leq n}\left\{\tau_{p q}, \eta_{p q}, \xi_{p q}\right\}, \\
& \varphi_{p}(t)=\varphi_{p}^{R}(t)+i \varphi_{p}^{I}(t)+j \varphi_{p}^{J}(t)+k \varphi_{p}^{K}(t),
\end{aligned}
$$

where $\varphi_{p}^{l} \in C\left(\left[t_{0}-\theta, t_{0}\right], \mathbb{R}\right), l \in \Upsilon$.

Based on $\left(H_{1}\right)$ and Hamilton's rules, one can obtain from (1) that

$$
\dot{x}_{p}^{R}(t)=-a_{p}(t) x_{p}^{R}(t)+\sum_{q=1}^{n}\left(b_{p q}^{R}(t) \tilde{f}_{q}^{R}[t, x, \tau]-b_{p q}^{I}(t) \tilde{f}_{q}^{I}[t, x, \tau]\right.
$$


Li et al. Advances in Difference Equations

(2019) 2019:63

Page 5 of 23

$$
\begin{aligned}
& \left.-b_{p q}^{J}(t) \tilde{f}_{q}^{J}[t, x, \tau]-b_{p q}^{K}(t) \tilde{f}_{q}^{K}[t, x, \tau]\right)+\sum_{q=1}^{n}\left(d_{p q}^{R}(t) \mu_{q}^{R}(t)\right. \\
& \left.-d_{p q}^{I}(t) \mu_{q}^{I}(t)-d_{p q}^{J}(t) \mu_{q}^{J}(t)-d_{p q}^{K}(t) \mu_{q}^{K}(t)\right) \\
& +\bigwedge_{q=1}^{n}\left(\alpha_{p q}^{R}(t) \int_{t-\eta_{p q}(t)}^{t} \tilde{g}_{q}^{R}[s, x] d s\right. \\
& -\alpha_{p q}^{I}(t) \int_{t-\eta_{p q}(t)}^{t} \tilde{g}_{q}^{I}[s, x] d s-\alpha_{p q}^{J}(t) \int_{t-\eta_{p q}(t)}^{t} \tilde{g}_{q}^{J}[s, x] d s \\
& \left.-\alpha_{p q}^{K}(t) \int_{t-\eta_{p q}(t)}^{t} \tilde{g}_{q}^{K}[s, x] d s\right)+\bigvee_{q=1}^{n}\left(\beta_{p q}^{R}(t) \int_{t-\xi_{p q}(t)}^{t} \tilde{g}_{q}^{R}[s, x] d s\right. \\
& -\beta_{p q}^{I}(t) \int_{t-\xi_{p q}(t)}^{t} \tilde{g}_{q}^{I}[s, x] d s-\beta_{p q}^{J}(t) \int_{t-\xi_{p q}(t)}^{t} \tilde{g}_{q}^{J}[s, x] d s \\
& \left.-\beta_{p q}^{K}(t) \int_{t-\xi_{p q}(t)}^{t} \tilde{g}_{q}^{K}[s, x] d s\right)+\bigwedge_{q=1}^{n}\left(T_{p q}^{R}(t) \mu_{q}^{R}(t)-T_{p q}^{I}(t) \mu_{q}^{I}(t)\right. \\
& \left.-T_{p q}^{J}(t) \mu_{q}^{J}(t)-T_{p q}^{K}(t) \mu_{q}^{K}(t)\right)+\bigvee_{q=1}^{n}\left(S_{p q}^{R}(t) \mu_{q}^{R}(t)-S_{p q}^{I}(t) \mu_{q}^{I}(t)\right. \\
& \left.-S_{p q}^{J}(t) \mu_{q}^{J}(t)-S_{p q}^{K}(t) \mu_{q}^{K}(t)\right)+I_{p}^{R}(t) \triangleq F_{p R}(t, x(t)), \quad p=1,2, \ldots, n, \\
& \dot{x}_{p}^{I}(t)=-a_{p}(t) x_{p}^{I}(t)+\sum_{q=1}^{n}\left(b_{p q}^{I}(t) \tilde{f}_{q}^{R}[t, x, \tau]+b_{p q}^{R}(t) \tilde{f}_{q}^{I}[t, x, \tau]\right. \\
& \left.-b_{p q}^{K}(t) \tilde{f}_{q}^{J}[t, x, \tau]+b_{p q}^{J}(t) \tilde{f}_{q}^{K}[t, x, \tau]\right)+\sum_{q=1}^{n}\left(d_{p q}^{I}(t) \mu_{q}^{R}(t)\right. \\
& \left.+d_{p q}^{R}(t) \mu_{q}^{I}(t)-d_{p q}^{K}(t) \mu_{q}^{J}(t)+d_{p q}^{J}(t) \mu_{q}^{K}(t)\right) \\
& +\bigwedge_{q=1}^{n}\left(\alpha_{p q}^{I}(t) \int_{t-\eta_{p q}(t)}^{t} \tilde{g}_{q}^{R}[s, x] d s\right. \\
& +\alpha_{p q}^{R}(t) \int_{t-\eta_{p q}(t)}^{t} \tilde{g}_{q}^{I}[s, x] d s-\alpha_{p q}^{K}(t) \int_{t-\eta_{p q}(t)}^{t} \tilde{g}_{q}^{J}[s, x] d s \\
& \left.+\alpha_{p q}^{J}(t) \int_{t-\eta_{p q}(t)}^{t} \tilde{g}_{q}^{K}[s, x] d s\right)+\bigvee_{q=1}^{n}\left(\beta_{p q}^{I}(t) \int_{t-\xi p q}^{t} \tilde{g}_{q}^{R}[s, x] d s\right. \\
& +\beta_{p q}^{R}(t) \int_{t-\xi_{p q}(t)}^{t} \tilde{g}_{q}^{I}[s, x] d s-\beta_{p q}^{K}(t) \int_{t-\xi_{p q}(t)}^{t} \tilde{g}_{q}^{J}[s, x] d s \\
& \left.+\beta_{p q}^{J}(t) \int_{t-\xi_{p q}(t)}^{t} \tilde{g}_{q}^{K}[s, x] d s\right)+\bigwedge_{q=1}^{n}\left(T_{p q}^{I}(t) \mu_{q}^{R}(t)+T_{p q}^{R}(t) \mu_{q}^{I}(t)\right. \\
& \left.-T_{p q}^{K}(t) \mu_{q}^{J}(t)+T_{p q}^{J}(t) \mu_{q}^{K}(t)\right)+\bigvee_{q=1}^{n}\left(S_{p q}^{I}(t) \mu_{q}^{R}(t)+S_{p q}^{R}(t) \mu_{q}^{I}(t)\right. \\
& \left.-S_{p q}^{K}(t) \mu_{q}^{J}(t)+S_{p q}^{J}(t) \mu_{q}^{K}(t)\right)+I_{p}^{I}(t) \triangleq F_{p I}(t, x(t)), \quad p=1,2, \ldots, n,
\end{aligned}
$$


Li et al. Advances in Difference Equations

(2019) 2019:63

Page 6 of 23

$$
\begin{aligned}
& \dot{x}_{p}^{J}(t)=-a_{p}(t) x_{p}^{J}(t)+\sum_{q=1}^{n}\left(b_{p q}^{J}(t) \tilde{f}_{q}^{R}[t, x, \tau]+b_{p q}^{K}(t) \tilde{f}_{q}^{I}[t, x, \tau]\right. \\
& \left.+b_{p q}^{R}(t) \tilde{f}_{q}^{J}[t, x, \tau]-b_{p q}^{I}(t) \tilde{f}_{q}^{K}[t, x, \tau]\right)+\sum_{q=1}^{n}\left(d_{p q}^{J}(t) \mu_{q}^{R}(t)\right. \\
& \left.+d_{p q}^{K}(t) \mu_{q}^{I}(t)+d_{p q}^{R}(t) \mu_{q}^{J}(t)-d_{p q}^{I}(t) \mu_{q}^{K}(t)\right) \\
& +\bigwedge_{q=1}^{n}\left(\alpha_{p q}^{J}(t) \int_{t-\eta_{p q}(t)}^{t} \tilde{g}_{q}^{R}[s, x] d s\right. \\
& +\alpha_{p q}^{K}(t) \int_{t-\eta_{p q}(t)}^{t} \tilde{g}_{q}^{I}[s, x] d s+\alpha_{p q}^{R}(t) \int_{t-\eta_{p q}(t)}^{t} \tilde{g}_{q}^{J}[s, x] d s \\
& \left.-\alpha_{p q}^{I}(t) \int_{t-\eta_{p q}(t)}^{t} \tilde{g}_{q}^{K}[s, x] d s\right)+\bigvee_{q=1}^{n}\left(\beta_{p q}^{J}(t) \int_{t-\xi p q}^{t} \tilde{g}_{q}^{R}[s, x] d s\right. \\
& +\beta_{p q}^{K}(t) \int_{t-\xi_{p q}(t)}^{t} \tilde{g}_{q}^{I}[s, x] d s+\beta_{p q}^{R}(t) \int_{t-\xi_{p q}(t)}^{t} \tilde{g}_{q}^{J}[s, x] d s \\
& \left.-\beta_{p q}^{I}(t) \int_{t-\xi_{p q}(t)}^{t} \tilde{g}_{q}^{K}[s, x] d s\right)+\bigwedge_{q=1}^{n}\left(T_{p q}^{J}(t) \mu_{q}^{R}(t)+T_{p q}^{K}(t) \mu_{q}^{I}(t)\right. \\
& \left.+T_{p q}^{R}(t) \mu_{q}^{J}(t)-T_{p q}^{I}(t) \mu_{q}^{K}(t)\right)+\bigvee_{q=1}^{n}\left(S_{p q}^{J}(t) \mu_{q}^{R}(t)+S_{p q}^{K}(t) \mu_{q}^{I}(t)\right. \\
& \left.+S_{p q}^{R}(t) \mu_{q}^{J}(t)-S_{p q}^{I}(t) \mu_{q}^{K}(t)\right)+I_{p}^{J}(t) \triangleq F_{p J}(t, x(t)), \quad p=1,2, \ldots, n, \\
& \dot{x}_{p}^{K}(t)=-a_{p}(t) x_{p}^{K}(t)+\sum_{q=1}^{n}\left(b_{p q}^{K}(t) \tilde{f}_{q}^{R}[t, x, \tau]-b_{p q}^{J}(t) \tilde{f}_{q}^{I}[t, x, \tau]\right. \\
& \left.+b_{p q}^{I}(t) \tilde{f}_{q}^{J}[t, x, \tau]+b_{p q}^{R}(t) \tilde{f}_{q}^{K}[t, x, \tau]\right)+\sum_{q=1}^{n}\left(d_{p q}^{K}(t) \mu_{q}^{R}(t)\right. \\
& \left.-d_{p q}^{J}(t) \mu_{q}^{I}(t)+d_{p q}^{I}(t) \mu_{q}^{J}(t)+d_{p q}^{R}(t) \mu_{q}^{K}(t)\right) \\
& +\bigwedge_{q=1}^{n}\left(\alpha_{p q}^{K}(t) \int_{t-\eta_{p q}(t)}^{t} \tilde{g}_{q}^{R}[s, x] d s\right. \\
& -\alpha_{p q}^{J}(t) \int_{t-\eta_{p q}(t)}^{t} \tilde{g}_{q}^{I}[s, x] d s+\alpha_{p q}^{I}(t) \int_{t-\eta_{p q}(t)}^{t} \tilde{g}_{q}^{J}[s, x] d s \\
& \left.+\alpha_{p q}^{R}(t) \int_{t-\eta_{p q}(t)}^{t} \tilde{g}_{q}^{K}[s, x] d s\right)+\bigvee_{q=1}^{n}\left(\beta_{p q}^{K}(t) \int_{t-\xi_{p q}(t)}^{t} \tilde{g}_{q}^{R}[s, x] d s\right. \\
& -\beta_{p q}^{J}(t) \int_{t-\xi_{p q}(t)}^{t} \tilde{g}_{q}^{I}[s, x] d s+\beta_{p q}^{I}(t) \int_{t-\xi_{p q}(t)}^{t} \tilde{g}_{q}^{J}[s, x] d s \\
& \left.+\beta_{p q}^{R}(t) \int_{t-\xi_{p q}(t)}^{t} \tilde{g}_{q}^{K}[s, x] d s\right)+\bigwedge_{q=1}^{n}\left(T_{p q}^{K}(t) \mu_{q}^{R}(t)-T_{p q}^{J}(t) \mu_{q}^{I}(t)\right. \\
& \left.+T_{p q}^{I}(t) \mu_{q}^{J}(t)+T_{p q}^{R}(t) \mu_{q}^{K}(t)\right)+\bigvee_{q=1}^{n}\left(S_{p q}^{K}(t) \mu_{q}^{R}(t)-S_{p q}^{J}(t) \mu_{q}^{I}(t)\right.
\end{aligned}
$$




$$
\left.+S_{p q}^{I}(t) \mu_{q}^{J}(t)+S_{p q}^{R}(t) \mu_{q}^{K}(t)\right)+I_{p}^{K}(t) \triangleq F_{p K}(t, x(t)), \quad p=1,2, \ldots, n
$$

where $x_{p}^{R}(t)+i x_{p}^{I}(t)+j x_{p}^{J}(t)+k x_{p}^{K}(t)=x_{p}(t), \tilde{f}_{q}^{l}[t, x, \tau]$ and $\tilde{g}_{q}^{l}[s, x]$ denote $f_{q}^{l}\left(x_{q}^{R}\left(t-\tau_{p q}(t)\right)\right.$, $\left.x_{q}^{I}\left(t-\tau_{p q}(t)\right), x_{q}^{J}\left(t-\tau_{p q}(t)\right), x_{q}^{K}\left(t-\tau_{p q}(t)\right)\right)$, and $g_{q}^{l}\left(x_{q}^{R}(s), x_{q}^{I}(s), x_{q}^{J}(s), x_{q}^{K}(s)\right)$, respectively, $p, q=$ $1,2, \ldots, n, l \in \Upsilon$. That is, (1) can be expressed in the following form:

$$
\dot{x}_{p}^{l}(t)=F_{p_{l}}(t, x(t)), \quad p=1,2, \ldots, n, l \in \Upsilon,
$$

with the initial value

$$
x_{p}^{l}(s)=\varphi_{p}^{l}(s), \quad s \in\left[t_{0}-\theta, t_{0}\right], p=1,2, \ldots, n .
$$

Remark 1 If $x=\left(x_{1}^{R}, \ldots, x_{n}^{R}, x_{1}^{I}, \ldots, x_{n}^{I}, x_{1}^{J}, \ldots, x_{n}^{J}, x_{1}^{K}, \ldots, x_{n}^{K}\right)^{T}$ is a solution to system (2), then $u=\left(x_{1}, x_{2}, \ldots, x_{n}\right)^{T}$, where $x_{p}=x_{p}^{R}+i x_{p}^{I}+j x_{p}^{J}+k x_{p}^{K}, p=1,2, \ldots, n$, is a solution to (1). Thus, to study the existence and stability of solutions of (1), we just need to study the existence and stability of solutions of system (2).

Definition 2 Let $x=\left(x_{1}^{R}, \ldots, x_{n}^{R}, x_{1}^{I}, \ldots, x_{n}^{I}, x_{1}^{J}, \ldots, x_{n}^{J}, x_{1}^{K}, \ldots, x_{n}^{K}\right)^{T}$ be a solution of (2) with the initial value $\varphi=\left(\varphi_{1}^{R}, \ldots, \varphi_{n}^{R}, \varphi_{1}^{I}, \ldots, \varphi_{n}^{I}, \varphi_{1}^{I}, \ldots, \varphi_{n}^{I}, \varphi_{1}^{K}, \ldots, \varphi_{n}^{K}\right)^{T}$ and $y=\left(y_{1}^{R}, \ldots, y_{n}^{R}, y_{1}^{I}, \ldots\right.$, $\left.y_{n}^{I}, y_{1}^{J}, \ldots, y_{n}^{J}, y_{1}^{K}, \ldots, y_{n}^{K}\right)^{T}$ be an arbitrary solution of system (2) with the initial value $\varphi=$ $\left(\varphi_{1}^{R}, \ldots, \varphi_{n}^{R}, \varphi_{1}^{I}, \ldots, \varphi_{n}^{I}, \varphi_{1}^{J}, \ldots, \varphi_{n}^{J}, \varphi_{1}^{K}, \ldots, \varphi_{n}^{K}\right)^{T}$, respectively, where $\varphi, \psi \in C\left(\left[t_{0}-\theta, t_{0}\right], \mathbb{R}^{4 n}\right)$.

If there exist constants $\lambda>0$ and $M>0$ such that

$$
\|x(t)-y(t)\| \leq M\|\varphi-\psi\| e^{-\lambda\left(t-t_{0}\right)}, \quad t \geq t_{0}
$$

where

$$
\|\varphi-\psi\|=\max _{\substack{1 \leq q \leq n \\ l \in \Upsilon}}\left\{\sup _{\substack{s \in\left[t_{0}-\theta, t_{0}\right] \\ \rho_{p}}} \rho_{p}^{-1}\left|\varphi_{p}^{l}(s)-\psi_{p}^{l}(s)\right|\right\} .
$$

Then the solution $x$ of system (2) is said to be globally exponentially stable.

Lemma 2 ([25]) Let $\alpha_{p q}^{l}, \beta_{p q}^{l}, x_{q}^{R}, x_{q}^{I}, x_{q}^{J}, x_{q}^{K}, y_{q}^{R}, y_{q}^{I}, y_{q}^{J}, y_{q}^{K}: \mathbb{R} \rightarrow \mathbb{R}$ and $g_{q}^{r}: \mathbb{R}^{4} \rightarrow \mathbb{R}$ be continuous functions for $p, q=1,2, \ldots, n, l, r \in \Upsilon$, then we have

$$
\begin{aligned}
& \left|\bigwedge_{q=1}^{n} \alpha_{p q}^{l} g_{q}^{r}\left(x_{q}^{R}, x_{q}^{I}, x_{q}^{J}, x_{q}^{K}\right)-\bigwedge_{q=1}^{n} \alpha_{p q}^{l} g_{q}^{r}\left(y_{q}^{R}, y_{q}^{I}, y_{q}^{J}, y_{q}^{K}\right)\right| \\
& \leq \sum_{q=1}^{n}\left|\alpha_{p q}^{l} \| g_{q}^{r}\left(x_{q}^{R}, x_{q}^{I}, x_{q}^{J}, x_{q}^{K}\right)-g_{q}^{r}\left(y_{q}^{R}, y_{q}^{I}, y_{q}^{J}, y_{q}^{K}\right)\right|, \\
& \quad\left|\bigvee_{q=1}^{n} \beta_{p q}^{l} g_{q}^{r}\left(x_{q}^{R}, x_{q}^{I}, x_{q}^{J}, x_{q}^{K}\right)-\bigvee_{q=1}^{n} \beta_{p q}^{l} g_{q}^{r}\left(y_{q}^{R}, y_{q}^{I}, y_{q}^{J}, y_{q}^{K}\right)\right| \\
& \quad \leq \sum_{q=1}^{n}\left|\beta_{p q}^{l} \| g_{q}^{r}\left(x_{q}^{R}, x_{q}^{I}, x_{q}^{J}, x_{q}^{K}\right)-g_{q}^{r}\left(y_{q}^{R}, y_{q}^{I}, y_{q}^{J}, y_{q}^{K}\right)\right| .
\end{aligned}
$$




\section{Main results}

In this section, we establish some sufficient conditions for the existence and exponential stability of periodic solutions of (1).

Set

$$
\begin{aligned}
\mathbb{X}= & \left\{x=\left(x_{1}^{R}, x_{1}^{I}, x_{1}^{J}, x_{1}^{K}, \ldots, x_{n}^{R}, x_{n}^{I}, x_{n}^{J}, x_{n}^{K}\right)^{T} \in C\left(\mathbb{R}, \mathbb{R}^{4 n}\right),\right. \\
& x(t+\omega)=x(t), t \in \mathbb{R}\} .
\end{aligned}
$$

For constant vector $\rho=\left(\rho_{1_{R}}, \rho_{1_{I}}, \rho_{1_{J}}, \rho_{1_{K}}, \ldots, \rho_{n_{R}}, \rho_{n_{I}}, \rho_{n_{J}}, \rho_{n_{K}}\right)^{T} \in \mathbb{R}^{4 n}$ with $\rho_{p_{l}}>0$ for $p=$ $1,2, \ldots, n, l \in \Upsilon$, we define the following norm:

$$
\|x\|_{\mathbb{X}}=\max _{\substack{1 \leq p \leq n \\ l \in \Upsilon}}\left\{\sup _{t \in[0, \omega]} \rho_{p_{l}}^{-1}\left|x_{p}^{l}(t)\right|\right\} .
$$

Then $\mathbb{X}$ is a Banach space when it is endowed with the norm $\|\cdot\|_{\mathbb{X}}$.

Obviously, if $x=\left(x_{1}^{R}, x_{1}^{I}, x_{1}^{J}, x_{1}^{K}, \ldots, x_{n}^{R}, x_{n}^{I}, x_{n}^{J}, x_{n}^{K}\right)^{T} \in \mathbb{X}$ is a solution of system (2), then

$$
\begin{aligned}
\left(x_{p}^{l}(t) e^{\int_{t_{0}}^{t} a_{p}(s) d s}\right)^{\prime} & =\dot{x}_{p}^{l}(t) e^{\int_{t_{0}}^{t} a_{p}(s) d s}+a_{p}(t) x_{p}^{l}(t) e^{\int_{t_{0}}^{t} a_{p}(s) d s} \\
& =e^{\int_{t_{0}}^{t} a_{p}(s) d s}\left[F_{p_{l}}(t, x(t))+a_{p}(t) x_{p}^{l}(t)\right]
\end{aligned}
$$

for $p=1,2, \ldots, n, l \in \Upsilon$. Integrating both sides of (3) over $[t, t+\omega]$, we can get

$$
\begin{aligned}
x_{p}^{l}(t) & =\int_{t}^{t+\omega} \frac{e^{\int_{t_{0}}^{s} a_{p}(u) d u}}{e^{\int_{t_{0}}^{t+\omega} a_{p}(u) d u}-e^{\int_{t_{0}}^{t} a_{p}(u) d u}}\left[F_{p_{l}}(s, x(s))+a_{p}(s) x_{p}^{l}(s)\right] d s \\
& =\int_{t}^{t+\omega} \frac{e^{-\int_{s}^{t+\omega} a_{p}(u) d u}}{1-e^{-\omega \bar{a}_{p}}}\left[F_{p_{l}}(s, x(s))+a_{p}(s) x_{p}^{l}(s)\right] d s \\
& =\int_{t}^{t+\omega} G_{p}(t, s)\left[F_{p_{l}}(s, x(s))+a_{p}(s) x_{p}^{l}(s)\right] d s,
\end{aligned}
$$

where $G_{p}(t, s)=\frac{e^{-\int_{s}^{t+\omega} a_{p}(u) d u}}{1-e^{-\omega \bar{a}_{p}}}, t \leq s \leq t+\omega, p=1,2, \ldots, n, l \in \Upsilon$.

Define an operator $\Phi: \mathbb{X} \rightarrow \mathbb{X}$ by

$$
\Phi x=\left(\Phi x_{1}^{R}, \Phi x_{1}^{I}, \Phi x_{1}^{J}, \Phi x_{1}^{K}, \ldots, \Phi n_{n}^{R}, \Phi x_{n}^{I}, \Phi x_{n}^{J}, \Phi x_{n}^{K}\right)^{T}
$$

where for $p=1,2, \ldots, n, l \in \Upsilon$,

$$
(\Phi x)_{p}^{l}(t)=\int_{t}^{t+\omega} G_{p}(t, s)\left[F_{p_{l}}(s, x(s))+a_{p}(s) x_{p}^{l}(s)\right] d s, \quad t \in \mathbb{R} .
$$

From (4), it is easy to verify that $x \in \mathbb{X}$ is an $\omega$-periodic solution of system (2) provided $x$ is a fixed point of $\Phi$ in $\mathbb{X}$.

It is easy to see that if there exist positive numbers $\rho_{1_{R}}, \rho_{1_{I}}, \rho_{1_{j}}, \rho_{1_{K}}, \ldots, \rho_{n_{R}}, \rho_{n_{I}}, \rho_{n_{J}}, \rho_{n_{K}}$ such that

$$
\gamma=\max _{\substack{1 \leq p \leq n \\ l, r \in \Upsilon}} \sup _{\substack{t \in \mathbb{R} \\ \text { f }}}\left\{\rho _ { p _ { l } } ^ { - 1 } \int _ { t } ^ { t + \omega } G _ { p } ( t , s ) \left(\sum _ { q = 1 } ^ { n } \left(\left|b_{p q}^{R}(s)\right|+\left|b_{p q}^{I}(s)\right|+\left|b_{p q}^{J}(s)\right|\right.\right.\right.
$$


Li et al. Advances in Difference Equations

(2019) 2019:63

Page 9 of 23

$$
\begin{aligned}
& \left.+\left|b_{p q}^{K}(s)\right|\right) \rho_{q_{r}} L_{f}+\sum_{q=1}^{n}\left(\left|\alpha_{p q}^{R}(s)\right|+\left|\alpha_{p q}^{I}(s)\right|+\left|\alpha_{p q}^{J}(s)\right|+\left|\alpha_{p q}^{K}(s)\right|\right) \rho_{q_{r}} L_{g} \theta \\
& \left.\left.+\sum_{q=1}^{n}\left(\left|\beta_{p q}^{R}(s)\right|+\left|\beta_{p q}^{I}(s)\right|+\left|\beta_{p q}^{J}(s)\right|+\left|\beta_{p q}^{K}(s)\right|\right) \rho_{q_{r}} L_{g} \theta\right) d s\right\}<\frac{1}{4},
\end{aligned}
$$

then there exists a sufficiently large $\kappa \geq 1$ such that $\gamma \leq \frac{1}{4}\left(1-\iota \kappa^{-1}\right)$, where

$$
\iota=\max _{\substack{1 \leq p \leq n \\ l \in \Upsilon}}\left\{\frac{\omega\left(\sum_{q=1}^{n} 4\left(d_{p q} \mu_{q}+T_{p q} \mu_{q}+S_{p q} \mu_{q}\right)+I_{p}\right)}{\rho_{p_{l}}\left(1-e^{-\omega \bar{a}_{p}}\right)}\right\} .
$$

Lemma 3 Under hypotheses $\left(H_{1}\right)-\left(H_{3}\right)$, suppose that there exist positive numbers $\rho_{1_{R}}, \rho_{1_{I}}$, $\rho_{1_{J}}, \rho_{1_{K}}, \ldots, \rho_{n_{R}}, \rho_{n_{I}}, \rho_{n_{J}}, \rho_{n_{K}}$ such that inequality (7) holds, then the mapping $\Phi$ is a selfmapping in the region $\mathbb{B}=\left\{x=\left(x_{1}^{R}, x_{1}^{I}, x_{1}^{J}, x_{1}^{K}, \ldots, x_{n}^{R}, x_{n}^{I}, x_{n}^{J}, x_{n}^{K}\right)^{T} \in \mathbb{X}:\|x\| \leq \kappa\right\}$.

Proof For $x \in \mathbb{B}$, from (6), we have

$$
\begin{aligned}
& \left|\rho_{p_{R}}^{-1}(\Phi x)_{p}^{R}(t)\right| \\
& =\left|\rho_{p_{R}}^{-1} \int_{t}^{t+\omega} G_{p}(t, s)\left[F_{p R}(s, x(s))+a_{p}(s) x_{p}^{R}(s)\right] d s\right| \\
& =\mid \rho_{p_{R}}^{-1} \int_{t}^{t+\omega} G_{p}(t, s)\left\{\sum _ { q = 1 } ^ { n } \left(b_{p q}^{R}(s) \tilde{f}_{q}^{R}[s, x, \tau]-b_{p q}^{I}(s) \tilde{f}_{q}^{I}[s, x, \tau]\right.\right. \\
& \left.-b_{p q}^{J}(s) \tilde{f}_{q}^{J}[s, x, \tau]-b_{p q}^{K}(s) \tilde{f}_{q}^{K}[s, x, \tau]\right)+\sum_{q=1}^{n}\left(d_{p q}^{R}(s) \mu_{q}^{R}(s)\right. \\
& \left.-d_{p q}^{I}(s) \mu_{q}^{I}(s)-d_{p q}^{J}(s) \mu_{q}^{J}(s)-d_{p q}^{K}(s) \mu_{q}^{K}(s)\right) \\
& +\bigwedge_{q=1}^{n}\left(\alpha_{p q}^{R}(s) \int_{s-\eta_{p q}(s)}^{s} \tilde{g}_{q}^{R}[u, x] d u-\alpha_{p q}^{I}(s) \int_{s-\eta_{p q}(s)}^{s} \tilde{g}_{q}^{I}[u, x] d u\right. \\
& \left.-\alpha_{p q}^{J}(s) \int_{s-\eta_{p q}(s)}^{s} \tilde{g}_{q}^{J}[u, x] d u-\alpha_{p q}^{K}(s) \int_{s-\eta_{p q}(s)}^{s} \tilde{g}_{q}^{K}[u, x] d u\right) \\
& +\bigvee_{q=1}^{n}\left(\beta_{p q}^{R}(s) \int_{s-\xi_{p q}(s)}^{s} \tilde{g}_{q}^{R}[u, x] d u-\beta_{p q}^{I}(s) \int_{s-\xi_{p q}(s)}^{s} \tilde{g}_{q}^{I}[u, x] d u\right. \\
& \left.-\beta_{p q}^{J}(s) \int_{s-\xi p q}^{s} \tilde{g}_{q}^{J}[u, x] d u-\beta_{p q}^{K}(s) \int_{s-\xi_{p q}(s)}^{s} \tilde{g}_{q}^{K}[u, x] d u\right) \\
& +\bigwedge_{q=1}^{n}\left(T_{p q}^{R}(s) \mu_{q}^{R}(s)-T_{p q}^{I}(s) \mu_{q}^{I}(s)-T_{p q}^{J}(s) \mu_{q}^{J}(s)-T_{p q}^{K}(s) \mu_{q}^{K}(s)\right) \\
& \left.+\bigvee_{q=1}^{n}\left(S_{p q}^{R}(s) \mu_{q}^{R}(s)-S_{p q}^{I}(s) \mu_{q}^{I}(s)-S_{p q}^{J}(s) \mu_{q}^{J}(s)-S_{p q}^{K}(s) \mu_{q}^{K}(s)\right)+I_{p}^{R}(s)\right\} d s \mid \\
& \leq \rho_{p_{R}}^{-1} \int_{t}^{t+\omega} G_{p}(t, s)\left\{\sum _ { q = 1 } ^ { n } \left(\left|b_{p q}^{R}(s)\right|\left|\tilde{f}_{q}^{R}[s, x, \tau]-\tilde{f}_{q}^{R}[0]\right|\right.\right. \\
& +\left|b_{p q}^{I}(s)\right|\left|\tilde{f}_{q}^{I}[s, x, \tau]-\tilde{f}_{q}^{I}[0]\right|+\left|b_{p q}^{J}(s)\right|\left|\tilde{f}_{q}^{J}[s, x, \tau]-\tilde{f}_{q}^{J}[0]\right|
\end{aligned}
$$


Li et al. Advances in Difference Equations

(2019) 2019:63

Page 10 of 23

$$
\begin{aligned}
& \left.+\left|b_{p q}^{K}(s)\right|\left|\tilde{f}_{q}^{K}[s, x, \tau]-\tilde{f}_{q}^{K}[0]\right|\right) \\
& +\left|\bigwedge_{q=1}^{n} \alpha_{p q}^{R}(s) \int_{s-\eta_{p q}(s)}^{s} \tilde{g}_{q}^{R}[u, x] d u-\bigwedge_{q=1}^{n} \alpha_{p q}^{R}(s) \int_{s-\eta_{p q}(s)}^{s} \tilde{g}_{q}^{R}[0] d u\right| \\
& +\left|\bigwedge_{q=1}^{n} \alpha_{p q}^{I}(s) \int_{s-\eta_{p q}(s)}^{s} \tilde{g}_{q}^{I}[u, x] d u-\bigwedge_{q=1}^{n} \alpha_{p q}^{I}(s) \int_{s-\eta_{p q}(s)}^{s} \tilde{g}_{q}^{I}[0] d u\right| \\
& +\left|\bigwedge_{q=1}^{n} \alpha_{p q}^{J}(s) \int_{s-\eta_{p q}(s)}^{s} \tilde{g}_{q}^{J}[u, x] d u-\bigwedge_{q=1}^{n} \alpha_{p q}^{J}(s) \int_{s-\eta_{p q}(t)}^{t} \tilde{g}_{q}^{J}[0] d u\right| \\
& +\left|\bigwedge_{q=1}^{n} \alpha_{p q}^{K}(s) \int_{s-\eta_{p q}(s)}^{s} \tilde{g}_{q}^{K}[u, x] d u-\bigwedge_{q=1}^{n} \alpha_{p q}^{K}(s) \int_{s-\eta_{p q}(s)}^{s} \tilde{g}_{q}^{K}[0] d u\right| \\
& +\left|\bigvee_{q=1}^{n} \beta_{p q}^{R}(s) \int_{s-\xi_{p q}(s)}^{s} \tilde{g}_{q}^{R}[u, x] d u-\bigvee_{q=1}^{n} \beta_{p q}^{R}(s) \int_{s-\xi_{p q}(s)}^{s} \tilde{g}_{q}^{R}[0] d u\right| \\
& +\left|\bigvee_{q=1}^{n} \beta_{p q}^{I}(s) \int_{s-\xi_{p q}(s)}^{s} \tilde{g}_{q}^{I}[u, x] d u-\bigvee_{q=1}^{n} \beta_{p q}^{I}(s) \int_{s-\xi_{p q}(s)}^{s} \tilde{g}_{q}^{I}[0] d u\right| \\
& +\left|\bigvee_{q=1}^{n} \beta_{p q}^{J}(s) \int_{s-\xi_{p q}(s)}^{s} \tilde{g}_{q}^{J}[u, x] d u-\bigvee_{q=1}^{n} \beta_{p q}^{J}(s) \int_{s-\xi_{p q}(s)}^{s} \tilde{g}_{q}^{J}[0] d u\right| \\
& \left.+\left|\bigvee_{q=1}^{n} \beta_{p q}^{K}(s) \int_{s-\xi_{p q}(s)}^{s} \tilde{g}_{q}^{K}[u, x] d u-\bigvee_{q=1}^{n} \beta_{p q}^{K}(s) \int_{s-\xi_{p q}(s)}^{t} \tilde{g}_{q}^{K}[0] d u\right|\right\} d s \\
& +\frac{\omega}{\rho_{p_{R}}\left(1-e^{\left.-\omega \bar{a}_{p}\right)}\right.}\left(\sum_{q=1}^{n} 4\left(d_{p q} \mu_{q}+T_{p q} \mu_{q}+S_{p q} \mu_{q}\right)+I_{p}\right) \\
& \leq \rho_{p_{R}}^{-1} \int_{t}^{t+\omega} G_{p}(t, s)\left\{\sum_{q=1}^{n}\left(\left|b_{p q}^{R}(s)\right|+\left|b_{p q}^{I}(s)\right|+\left|b_{p q}^{J}(s)\right|+\left|b_{p q}^{K}(s)\right|\right) L_{f}\right. \\
& \times\left(\left|x_{q}^{R}\left(s-\tau_{p q}(s)\right)\right|+x_{q}^{I}\left(s-\tau_{p q}(s)\right)\left|+x_{q}^{J}\left(s-\tau_{p q}(s)\right)\right|+x_{q}^{K}\left(s-\tau_{p q}(s)\right) \mid\right) \\
& +\sum_{q=1}^{n}\left(\left|\alpha_{p q}^{R}(s)\right|+\left|\alpha_{p q}^{I}(s)\right|+\left|\alpha_{p q}^{J}(s)\right|+\left|\alpha_{p q}^{K}(s)\right|\right) L_{g} \int_{s-\eta_{p q}(s)}^{s}\left(\left|x_{q}^{R}(u)\right|\right. \\
& \left.+x_{q}^{I}(u)\left|+x_{q}^{J}(u)\right|+x_{q}^{K}(u) \mid\right) d u+\sum_{q=1}^{n}\left(\left|\beta_{p q}^{R}(s)\right|+\left|\beta_{p q}^{I}(s)\right|+\left|\beta_{p q}^{J}(s)\right|\right. \\
& \left.\left.+\left|\beta_{p q}^{K}(s)\right|\right) L_{g} \int_{s-\xi_{p q}(s)}^{s}\left(\left|x_{q}^{R}(u)\right|+x_{q}^{I}(u)\left|+x_{q}^{J}(u)\right|+x_{q}^{K}(u) \mid\right) d u\right\} d s \\
& +\frac{\omega\left(\sum_{q=1}^{n} 4\left(d_{p q} \mu_{q}+T_{p q} \mu_{q}+S_{p q} \mu_{q}\right)+I_{p}\right)}{\rho_{p_{R}}\left(1-e^{-\omega \bar{a}_{p}}\right)} \\
& \leq \rho_{p_{R}}^{-1} \int_{t}^{t+\omega} G_{p}(t, s)\left\{\sum _ { q = 1 } ^ { n } ( | b _ { p q } ^ { R } ( s ) | + | b _ { p q } ^ { I } ( s ) | + | b _ { p q } ^ { J } ( s ) | + | b _ { p q } ^ { K } ( s ) | ) \left(\rho_{q_{R}}+\rho_{q_{I}}\right.\right. \\
& \left.+\rho_{q_{J}}+\rho_{q_{K}}\right) L_{f}+\sum_{q=1}^{n}\left(\left|\alpha_{p q}^{R}(s)\right|+\left|\alpha_{p q}^{I}(s)\right|+\left|\alpha_{p q}^{J}(s)\right|+\left|\alpha_{p q}^{K}(s)\right|\right)\left(\rho_{q_{R}}+\rho_{q_{I}}\right.
\end{aligned}
$$




$$
\begin{aligned}
& \left.+\rho_{q_{J}}+\rho_{q_{K}}\right) L_{g} \theta+\sum_{q=1}^{n}\left(\left|\beta_{p q}^{R}(s)\right|+\left|\beta_{p q}^{I}(s)\right|+\left|\beta_{p q}^{J}(s)\right|+\left|\beta_{p q}^{K}(s)\right|\right)\left(\rho_{q_{R}}+\rho_{q_{I}}\right. \\
& \left.\left.+\rho_{q_{J}}+\rho_{q_{K}}\right) L_{g} \theta\right\}\|x\| d s+\frac{\omega\left(\sum_{q=1}^{n} 4\left(d_{p q} \mu_{q}+T_{p q} \mu_{q}+S_{p q} \mu_{q}\right)+I_{p}\right)}{\rho_{p_{R}}\left(1-e^{-\omega \bar{a}_{p}}\right)} \\
& \leq 4 \gamma \kappa+\iota \leq 4 \kappa \times \frac{1}{4}\left(1-\iota \kappa^{-1}\right)+\iota=\kappa, \quad p=1,2, \ldots, n .
\end{aligned}
$$

Similarly, from (6), we can obtain

$$
\left|\rho_{p_{l}}^{-1}(\Phi x)_{p}^{l}(t)\right| \leq \kappa, \quad p=1,2, \ldots, n, l=I, J, K
$$

Thus,

$$
\|\Phi x\|_{\mathbb{X}}=\max _{\substack{1 \leq p \leq n \\ l \in \Upsilon}}\left\{\sup _{t \in \mathbb{R}} \rho_{p_{l}}^{-1}\left|(\Phi x)_{p}^{l}(t)\right|\right\} \leq \kappa
$$

The proof is complete.

Lemma 4 Assume that $\left(H_{1}\right)-\left(H_{3}\right)$ hold and suppose further that there exist positive numbers $\rho_{1_{R}}, \rho_{1_{I}}, \rho_{1_{J}}, \rho_{1_{K}}, \ldots, \rho_{n_{R}}, \rho_{n_{I}}, \rho_{n_{J}}, \rho_{n_{K}}$ such that inequality (7) holds, then the mapping $\Phi: \mathbb{B} \rightarrow \mathbb{B}$ defined by (5) is completely continuous.

Proof It is obvious that $\Phi$ is continuous.

Next, we prove that $\Phi$ is compact. For any $M>0$, let $S=\left\{x \in \mathbb{X}:\|x\|_{\mathbb{X}} \leq M\right\}$. Then, for $x \in S$, we have

$$
\begin{aligned}
& \left|f_{q}^{l}\left(x_{q}^{R}(s), x_{q}^{I}(s), x_{q}^{J}(s), x_{q}^{K}(s)\right)\right| \leq\left(\rho_{q_{R}}+\rho_{q_{I}}+\rho_{q_{J}}+\rho_{q_{K}}\right) L_{f} M, \\
& \left|g_{q}^{l}\left(x_{q}^{R}(s), x_{q}^{I}(s), x_{q}^{J}(s), x_{q}^{K}(s)\right)\right| \leq\left(\rho_{q_{R}}+\rho_{q_{I}}+\rho_{q_{J}}+\rho_{q_{K}}\right) L_{g} M .
\end{aligned}
$$

For $\forall x \in S$, we have

$$
\begin{aligned}
& \left|\rho_{p_{l}}^{-1}(\Phi x)_{p}^{l}(t)\right| \\
& \leq \rho_{p_{l}}^{-1} \int_{t}^{t+\omega} G_{p}(t, s)\left\{\sum _ { q = 1 } ^ { n } ( | b _ { p q } ^ { R } ( s ) | + | b _ { p q } ^ { I } ( s ) | + | b _ { p q } ^ { I } ( s ) | + | b _ { p q } ^ { K } ( s ) | ) \left(\rho_{q_{R}}+\rho_{q_{I}}\right.\right. \\
& \left.\quad+\rho_{q_{J}}+\rho_{q_{K}}\right) L_{f} M+\bigwedge_{q=1}^{n}\left(\left|\alpha_{p q}^{R}(s)\right|+\left|\alpha_{p q}^{I}(s)\right|+\left|\alpha_{p q}^{J}(s)\right|+\left|\alpha_{p q}^{K}(s)\right|\right)\left(\rho_{q_{R}}+\rho_{q_{I}}\right. \\
& \left.\quad+\rho_{q_{J}}+\rho_{q_{K}}\right) L_{g} M \theta+\bigvee_{q=1}^{n}\left(\left|\beta_{p q}^{R}(s)\right|+\left|\beta_{p q}^{I}(s)\right|+\left|\beta_{p q}^{I}(s)\right|+\left|\beta_{p q}^{K}(s)\right|\right)\left(\rho_{q_{R}}+\rho_{q_{I}}\right. \\
& \left.\left.\quad+\rho_{q_{J}}+\rho_{q_{K}}\right) L_{g} M \theta\right\} d s+\frac{\omega\left(\sum_{q=1}^{n} 4\left(d_{p q} \mu_{q}+T_{p q} \mu_{q}+S_{p q} \mu_{q}\right)+I_{p}\right)}{\rho_{p_{l}}\left(1-e^{-\omega \bar{a}_{p}}\right)} \\
& \leq \rho_{p_{l}}^{-1} \int_{t}^{t+\omega} G_{p}(t, s)\left\{\sum _ { q = 1 } ^ { n } ( | b _ { p q } ^ { R } ( s ) | + | b _ { p q } ^ { I } ( s ) | + | b _ { p q } ^ { I } ( s ) | + | b _ { p q } ^ { K } ( s ) | ) \left(\rho_{q_{R}}+\rho_{q_{I}}\right.\right.
\end{aligned}
$$




$$
\begin{aligned}
& \left.+\rho_{q_{J}}+\rho_{q_{K}}\right) L_{f} M+\sum_{q=1}^{n}\left(\left|\alpha_{p q}^{R}(s)\right|+\left|\alpha_{p q}^{I}(s)\right|+\left|\alpha_{p q}^{J}(s)\right|+\left|\alpha_{p q}^{K}(s)\right|\right)\left(\rho_{q_{R}}+\rho_{q_{I}}\right. \\
& \left.+\rho_{q_{J}}+\rho_{q_{K}}\right) L_{g} M \theta+\sum_{q=1}^{n}\left(\left|\beta_{p q}^{R}(s)\right|+\left|\beta_{p q}^{I}(s)\right|+\left|\beta_{p q}^{J}(s)\right|+\left|\beta_{p q}^{K}(s)\right|\right)\left(\rho_{q_{R}}+\rho_{q_{I}}\right. \\
& \left.\left.+\rho_{q_{J}}+\rho_{q_{K}}\right) L_{g} M \theta\right\} d s+\frac{\omega\left(\sum_{q=1}^{n} 4\left(d_{p q} \mu_{q}+T_{p q} \mu_{q}+S_{p q} \mu_{q}\right)+I_{p}\right)}{\rho_{p_{l}}\left(1-e^{-\omega \bar{a}_{p}}\right)} \\
& \leq \frac{\omega}{\rho_{p_{l}}\left(1-e^{-\omega \bar{a}_{p}}\right)} \sum_{q=1}^{n}\left[16 \rho^{+} M\left(b_{p q} L_{f}+\alpha_{p q} L_{g} \theta+\beta_{p q} L_{g} \theta\right)\right. \\
& \left.+4 \mu_{q}\left(d_{p q}+T_{p q}+S_{p q}\right)+I_{p}\right]:=C_{p l}, \quad p=1,2, \ldots, n, l \in \Upsilon .
\end{aligned}
$$

Therefore,

$$
\|\Phi x\|_{\mathbb{X}}=\max _{\substack{1 \leq p \leq n \\ l \in \Upsilon}}\left\{\sup _{t \in[0, \omega]} \rho_{p_{l}}^{-1}\left|(\Phi x)_{p}^{l}(t)\right|\right\} \leq \max _{\substack{1 \leq p \leq n \\ l \in \Upsilon}}\left\{C_{p l}\right\}, \quad \forall x \in S
$$

which implies that $\Phi S$ is uniformly bounded.

On the other hand,

$$
\begin{aligned}
{\left[(\Phi x)_{p}^{l}(t)\right]^{\prime}=} & {\left[\int_{t}^{t+\omega} G_{p}(t, s)\left[F_{p_{l}}(s, x(s))+a_{p}(s) x_{p}^{l}(s)\right] d s\right]^{\prime} } \\
= & \int_{t}^{t+\omega} \frac{\partial G_{p}(t, s)}{\partial t}\left[F_{p_{l}}(s, x(s))+a_{p}(s) x_{p}^{l}(s)\right] d s \\
& +G_{p}(t, t+\omega)\left[F_{p_{l}}(t+\omega, x(t+\omega))+a_{p}(t+\omega) x_{p}^{l}(t+\omega)\right] \\
& -G_{p}(t, t)\left[F_{p_{l}}(t, x(t))+a_{p}(t) x_{p}^{l}(t)\right] \\
= & -a_{p}(t)(\Phi x)_{p}^{l}(t)+F_{p_{l}}(t, x(t))+a_{p}(t) x_{p}^{l}(t), \quad p=1,2, \ldots, n, l \in \Upsilon .
\end{aligned}
$$

Hence, for $x \in S$, we have

$$
\begin{aligned}
\left|\rho_{p_{l}}^{-1}\left[(\Phi x)_{p}^{l}(t)\right]^{\prime}\right|= & \rho_{p_{l}}^{-1}\left|-a_{p}(t)(\Phi x)_{p}^{l}(t)+F_{p_{l}}(t, x(t))+a_{p}(t) x_{p}^{l}(t)\right| \\
\leq & \rho_{p_{l}}^{-1}\left|-a_{p}(t)(\Phi x)_{p}^{l}(t)\right|+\rho_{p_{l}}^{-1}\left|F_{p_{l}}(t, x(t))+a_{p}(t) x_{p}^{l}(t)\right| \\
\leq & a_{p}(t) C+\rho_{p_{l}}^{-1} \sum_{q=1}^{n}\left[16 \rho^{+} M\left(b_{p q} L_{f}+\alpha_{p q} L_{g}+\beta_{p q} L_{g}\right)\right. \\
& \left.+4 \mu_{q}\left(d_{p q}+T_{p q}+S_{p q}\right)+I_{p}\right]:=H_{p l}, \quad p=1,2, \ldots, n, l \in \Upsilon .
\end{aligned}
$$

Therefore,

$$
\left|(\Phi x)^{\prime}(t)\right| \leq \max _{\substack{1 \leq p \leq n \\ l \in \Upsilon}}\left\{\sup _{\substack{t \in[0, \omega] \\ \rho_{p_{l}}}} \rho^{-1}\left|\left[(\Phi x)_{p}^{l}(t)\right]^{\prime}\right|\right\} \leq \max _{\substack{1 \leq p \leq n \\ l \in \Upsilon}}\left\{H_{p l}\right\}, \quad \forall x \in S,
$$

which implies that $\Phi S$ is equi-continuous.

Hence, by the Ascoli-Arzela theorem, we know that the operator $\Phi$ is compact, and so it is completely continuous. The proof is complete. 
According to Lemma 1 and Remark 1, from Lemma 3 and Lemma 4, we have the following theorem.

Theorem 1 Assume that $\left(H_{1}\right)-\left(H_{3}\right)$ hold. Suppose further that there exist positive numbers $\rho_{1_{R}}, \rho_{1_{I}}, \rho_{1_{J}}, \rho_{1_{K}}, \ldots, \rho_{n_{R}}, \rho_{n_{I}}, \rho_{n_{J}}, \rho_{n_{K}}$ such that inequality (7) holds, then system (1) has at least one $\omega$-periodic solution.

Next, we will show the exponential stability of periodic solutions.

Theorem 2 Assume that $\left(H_{1}\right)-\left(H_{3}\right)$ hold. Furthermore, assume the following.

$\left(H_{4}\right)$ Functions $\tau_{p q}(t)$ are continuous differential functions and $\dot{\tau}_{p q}<1$, where $p, q=$ $1,2, \ldots, n$.

$\left(H_{5}\right)$ There exists a positive constant $\lambda>0$ satisfying

$$
\max _{1 \leq p \leq n}\left\{\lambda-a_{p}^{-}+\sum_{q=1}^{n} 16 e^{\lambda \theta}\left(\frac{b_{q p} L_{f}}{1-\dot{\tau}_{q p}}+\left(\alpha_{q p} \eta_{q p}+\beta_{q p} \xi_{q p}\right) L_{g}\right)\right\} \leq 0
$$

Then system (1) has a unique $\omega$-periodic solution and it is globally exponentially stable.

Proof From Theorem 1, we see that system (2) has an $\omega$-periodic solution $x(t)$. Suppose that the initial value of $x(t)$ is $\varphi(t)$. Let $y(t)$ be an arbitrary solution of system (2) with the initial value $\psi(t)$. Set $z_{p}^{l}(t)=x_{p}^{l}(t)-y_{p}^{l}(t)$, by $(2)$, for $l=R, p=1,2, \ldots, n$, we have

$$
\begin{aligned}
D^{+}\left(e^{\lambda\left(t-t_{0}\right)}\left|z_{p}^{R}(t)\right|\right) & \\
= & e^{\lambda\left(t-t_{0}\right)} D^{+}\left|z_{p}^{R}(t)\right|+\lambda e^{\lambda\left(t-t_{0}\right)}\left|z_{p}^{R}(t)\right| \\
= & \lambda e^{\lambda\left(t-t_{0}\right)}\left|z_{p}^{R}(t)\right|+e^{\lambda\left(t-t_{0}\right)} \operatorname{sgn}\left(z_{p}^{R}(t)\right) \dot{z}_{p}^{R}(t) \\
\leq & \lambda e^{\lambda\left(t-t_{0}\right)}\left|z_{p}^{R}(t)\right|+e^{\lambda\left(t-t_{0}\right)}\left\{-a_{p}^{-}\left|z_{p}^{R}(t)\right|+\sum_{q=1}^{n} 4 b_{p q} L_{f}\left(\left|z_{p}^{R}\left(t-\tau_{p q}(t)\right)\right|\right.\right. \\
& \left.+\left|z_{p}^{I}\left(t-\tau_{p q}(t)\right)\right|+\left|z_{p}^{J}\left(t-\tau_{p q}(t)\right)\right|+\left|z_{p}^{K}\left(t-\tau_{p q}(t)\right)\right|\right) \\
& +\bigwedge_{q=1}^{n} 4 \alpha_{p q} L_{g} \int_{t-\eta_{p q}(t)}^{t}\left(\left|z_{p}^{R}(s)\right|+\left|z_{p}^{I}(s)\right|+\left|z_{p}^{J}(s)\right|+\left|z_{p}^{K}(s)\right|\right) d s \\
& \left.+\bigvee_{q=1}^{n} 4 \beta_{p q} L_{g} \int_{t-\xi_{p q}(t)}^{t}\left(\left|z_{p}^{R}(s)\right|+\left|z_{p}^{I}(s)\right|+\left|z_{p}^{J}(s)\right|+\left|z_{p}^{K}(s)\right|\right) d s\right\} \\
\leq & \lambda e^{\lambda\left(t-t_{0}\right)}\left|z_{p}^{R}(t)\right|+e^{\lambda\left(t-t_{0}\right)}\left\{-a_{p}^{-}\left|z_{p}^{R}(t)\right|+\sum_{q=1}^{n} 4 b_{p q} L_{f}\left(\left|z_{p}^{R}\left(t-\tau_{p q}(t)\right)\right|\right.\right. \\
& \left.+\sum_{q=1}^{n} 4 \beta_{p q} L_{g} \int_{t-\xi_{p q}(t)}^{t}\left(\left|z_{p}^{R}(s)\right|+\left|z_{p}^{I}(s)\right|+\left|z_{p}^{J}(s)\right|+\left|z_{p}^{K}(s)\right|\right) d s\right\} . \\
& \left.+\left|z_{p}^{I}\left(t-\tau_{p q}(t)\right)\right|+\left|z_{p}^{J}\left(t-\tau_{p q}(t)\right)\right|+\left|z_{p}^{K}\left(t-\tau_{p q}(t)\right)\right|\right) \\
& +\sum_{q=1}^{n} 4 \alpha_{p q} L_{g} \int_{t-\eta_{p q}(t)}^{t}\left(\left|z_{p}^{R}(s)\right|+\left|z_{p}^{I}(s)\right|+\left|z_{p}^{J}(s)\right|+\left|z_{p}^{K}(s)\right|\right) d s \\
& \\
& \\
& \\
& \\
& \\
&
\end{aligned}
$$


Li et al. Advances in Difference Equations

(2019) 2019:63

Page 14 of 23

Repeating the above procession, for $p=1,2, \ldots, n, l=I, J, K$, we can obtain that

$$
\begin{aligned}
& D^{+}\left(e^{\lambda\left(t-t_{0}\right)}\left|z_{p}^{l}(t)\right|\right) \\
& \leq \quad \lambda e^{\lambda\left(t-t_{0}\right)}\left|z_{p}^{R}(t)\right|+e^{\lambda\left(t-t_{0}\right)}\left\{-a_{p}^{-}\left|z_{p}^{R}(t)\right|+\sum_{q=1}^{n} 4 b_{p q} L_{f}\left(\left|z_{p}^{R}\left(t-\tau_{p q}(t)\right)\right|\right.\right. \\
& \left.\quad+\left|z_{p}^{I}\left(t-\tau_{p q}(t)\right)\right|+\left|z_{p}^{J}\left(t-\tau_{p q}(t)\right)\right|+\left|z_{p}^{K}\left(t-\tau_{p q}(t)\right)\right|\right) \\
& \quad+\sum_{q=1}^{n} 4 \alpha_{p q} L_{g} \int_{t-\eta_{p q}(t)}^{t}\left(\left|z_{p}^{R}(s)\right|+\left|z_{p}^{I}(s)\right|+\left|z_{p}^{J}(s)\right|+\left|z_{p}^{K}(s)\right|\right) d s \\
& \left.\quad+\sum_{q=1}^{n} 4 \beta_{p q} L_{g} \int_{t-\xi_{p q}(t)}^{t}\left(\left|z_{p}^{R}(s)\right|+\left|z_{p}^{I}(s)\right|+\left|z_{p}^{J}(s)\right|+\left|z_{p}^{K}(s)\right|\right) d s\right\} .
\end{aligned}
$$

Define a Lyapunov function as follows:

$$
V(t)=V_{R}(t)+V_{I}(t)+V_{J}(t)+V_{K}(t)
$$

where, for $l \in \Upsilon$,

$$
\begin{aligned}
V_{l}(t)= & \sum_{p=1}^{n}\left\{\rho_{p_{l}}^{-1} e^{\lambda\left(t-t_{0}\right)}\left|z_{p}^{l}(t)\right|+\sum_{q=1}^{n} \frac{4 b_{p q} L_{f} \rho_{p_{l}}^{-1} e^{\lambda\left(\theta-t_{0}\right)}}{1-\dot{\tau}_{p q}}\right. \\
& \times \int_{t-\tau_{p q}(t)}^{t} e^{\lambda s}\left(\left|z_{q}^{R}(s)\right|+\left|z_{q}^{I}(s)\right|+\left|z_{q}^{J}(s)\right|+\left|z_{q}^{K}(s)\right|\right) d s \\
& +\sum_{q=1}^{n} 4 \alpha_{p q} L_{g} \rho_{p_{l}}^{-1} e^{\lambda\left(\theta-t_{0}\right)} \int_{-\eta_{p q}}^{0} \int_{t+u}^{t} e^{\lambda s}\left(\left|z_{q}^{R}(s)\right|+\left|z_{q}^{I}(s)\right|\right. \\
& \left.+\left|z_{q}^{J}(s)\right|+\left|z_{q}^{K}(s)\right|\right) d s d u+\sum_{q=1}^{n} 4 \beta_{p q} L_{g} \rho_{p_{l}}^{-1} e^{\lambda\left(\theta-t_{0}\right)} \\
& \left.\times \int_{-\xi_{p q}}^{0} \int_{t+u}^{t} e^{\lambda s}\left(\left|z_{q}^{R}(s)\right|+\left|z_{q}^{I}(s)\right|+\left|z_{q}^{J}(s)\right|+\left|z_{q}^{K}(s)\right|\right) d s d u\right\} .
\end{aligned}
$$

Now we calculate the time derivative of $V(t)$ along the trajectories of system (2), we get

$$
\begin{aligned}
D^{+} V_{l}(t)= & \sum_{p=1}^{n}\left\{\rho_{p_{l}}^{-1} D^{+}\left(e^{\lambda\left(t-t_{0}\right)}\left|z_{p}^{l}(t)\right|\right)+\sum_{q=1}^{n} \frac{4 b_{p q} L_{f} \rho_{p_{l}}^{-1} e^{\lambda\left(\theta-t_{0}\right)}}{1-\dot{\tau}_{p q}}\right. \\
& \times e^{\lambda t}\left(\left|z_{q}^{R}(t)\right|+\left|z_{q}^{I}(t)\right|+\left|z_{q}^{J}(t)\right|+\left|z_{q}^{J}(t)\right|\right) \\
& -\sum_{q=1}^{n} \frac{4 b_{p q} L_{f} \rho_{p l}^{-1} e^{\lambda\left(\theta-t_{0}\right)}\left(1-\dot{\tau}_{p q}(t)\right)}{1-\dot{\tau}_{p q}} e^{\lambda\left(t-\tau_{p q}(t)\right)}\left(\left|z_{q}^{R}\left(t-\tau_{p q}(t)\right)\right|\right. \\
& \left.+\left|z_{q}^{I}\left(t-\tau_{p q}(t)\right)\right|+\left|z_{q}^{J}\left(t-\tau_{p q}(t)\right)\right|+\left|z_{q}^{K}\left(t-\tau_{p q}(t)\right)\right|\right) \\
& +\sum_{q=1}^{n} 4 \alpha_{p q} L_{g} \rho_{p_{l}}^{-1} e^{\lambda\left(\theta-t_{0}\right)} \int_{-\eta_{p q}}^{0} e^{\lambda t}\left(\left|z_{q}^{R}(t)\right|+\left|z_{q}^{I}(t)\right|+\left|z_{q}^{J}(t)\right|\right.
\end{aligned}
$$




$$
\begin{aligned}
& \left.+\left|z_{q}^{K}(t)\right|\right) d u-\sum_{q=1}^{n} 4 \alpha_{p q} L_{g} \rho_{p_{l}}^{-1} e^{\lambda\left(\theta-t_{0}\right)} \int_{-\eta_{p q}}^{0} e^{\lambda(t+u)}\left(\left|z_{q}^{R}(t+u)\right|\right. \\
& \left.+\left|z_{q}^{I}(t+u)\right|+\left|z_{q}^{J}(t+u)\right|+\left|z_{q}^{K}(t+u)\right|\right) d u \\
& +\sum_{q=1}^{n} 4 \beta_{p q} L_{g} \rho_{p_{l}}^{-1} e^{\lambda\left(\theta-t_{0}\right)} \int_{-\xi_{p q}}^{0} e^{\lambda t}\left(\left|z_{q}^{R}(t)\right|+\left|z_{q}^{I}(t)\right|+\left|z_{q}^{J}(t)\right|\right. \\
& \left.+\left|z_{q}^{K}(t)\right|\right) d u-\sum_{q=1}^{n} 4 \beta_{p q} L_{g} \rho_{p_{l}}^{-1} e^{\lambda\left(\theta-t_{0}\right)} \int_{-\xi_{p q}}^{0} e^{\lambda(t+u)}\left(\left|z_{q}^{R}(t+u)\right|\right. \\
& \left.\left.+\left|z_{q}^{I}(t+u)\right|+\left|z_{q}^{J}(t+u)\right|+\left|z_{q}^{K}(t+u)\right|\right) d u\right\} \\
& \leq \sum_{p=1}^{n}\left\{\lambda \rho_{p_{l}}^{-1} e^{\lambda\left(t-t_{0}\right)}\left|z_{p}^{l}(t)\right|-a_{p}^{-} \rho_{p_{l}}^{-1} e^{\lambda\left(t-t_{0}\right)}\left|z_{p}^{l}(t)\right|\right. \\
& +\sum_{q=1}^{n} \frac{4 b_{p q} L_{f} \rho_{p_{l}}^{-1} e^{\lambda\left(\theta-t_{0}\right)}}{1-\dot{\tau}_{p q}} e^{\lambda t}\left(\left|z_{q}^{R}(t)\right|+\left|z_{q}^{I}(t)\right|+\left|z_{q}^{J}(t)\right|+\left|z_{q}^{J}(t)\right|\right) \\
& +\sum_{p=1}^{n} 4 b_{p q} L_{f} \rho_{p_{l}}^{-1} e^{\lambda\left(t-t_{0}\right)}\left(1-\frac{e^{\lambda\left(\theta-\tau_{p q}(t)\right)}\left(1-\dot{\tau}_{p q}(t)\right)}{1-\dot{\tau}_{p q}}\right) \\
& \times\left(\left|z_{q}^{R}\left(t-\tau_{p q}(t)\right)\right|+\left|z_{q}^{I}\left(t-\tau_{p q}(t)\right)\right|+\left|z_{q}^{J}\left(t-\tau_{p q}(t)\right)\right|\right. \\
& \left.+\left|z_{q}^{K}\left(t-\tau_{p q}(t)\right)\right|\right)+\sum_{q=1}^{n} 4 \alpha_{p q} L_{g} \rho_{p_{l}}^{-1} e^{\lambda\left(\theta-t_{0}\right)} \int_{-\eta_{p q}}^{0} e^{\lambda t}\left(\left|z_{q}^{R}(t)\right|\right. \\
& \left.+\left|z_{q}^{I}(t)\right|+\left|z_{q}^{J}(t)\right|+\left|z_{q}^{K}(t)\right|\right) d u+\sum_{q=1}^{n} 4 \alpha_{p q} L_{g} \rho_{p l}^{-1} e^{\lambda\left(t-t_{0}\right)} \\
& \times\left(1-e^{\lambda\left(\theta-\eta_{p q}\right)}\right) \int_{t-\eta_{p q}}^{t}\left(\left|z_{q}^{R}(s)\right|+\left|z_{q}^{I}(s)\right|+\left|z_{q}^{J}(s)\right|+\left|z_{q}^{K}(s)\right|\right) d s \\
& +\sum_{q=1}^{n} 4 \beta_{p q} L_{g} \rho_{p_{l}}^{-1} e^{\lambda\left(\theta-t_{0}\right)} \int_{-\xi_{p q}}^{0} e^{\lambda t}\left(\left|z_{q}^{R}(t)\right|+\left|z_{q}^{I}(t)\right|+\left|z_{q}^{J}(t)\right|\right. \\
& \left.+\left|z_{q}^{K}(t)\right|\right) d u+\sum_{q=1}^{n} 4 \beta_{p q} L_{g} \rho_{p l}^{-1} e^{\lambda\left(t-t_{0}\right)}\left(1-e^{\lambda\left(\theta-\xi_{p q}\right)}\right) \\
& \left.\times \int_{t-\xi_{p q}}^{t}\left(\left|z_{q}^{R}(s)\right|+\left|z_{q}^{I}(s)\right|+\left|z_{q}^{J}(s)\right|+\left|z_{q}^{K}(s)\right|\right) d s\right\} \\
& \leq \sum_{p=1}^{n}\left\{\lambda \rho_{p_{l}}^{-1} e^{\lambda\left(t-t_{0}\right)}\left|z_{p}^{l}(t)\right|-a_{p}^{-} \rho_{p_{l}}^{-1} e^{\lambda\left(t-t_{0}\right)}\left|z_{p}^{l}(t)\right|\right. \\
& +\sum_{q=1}^{n} \frac{4 b_{p q} L_{f} \rho_{p_{l}}^{-1} e^{\lambda\left(\theta-t_{0}\right)}}{1-\dot{\tau}_{p q}} e^{\lambda t}\left(\left|z_{q}^{R}(t)\right|+\left|z_{q}^{I}(t)\right|+\left|z_{q}^{J}(t)\right|+\left|z_{q}^{J}(t)\right|\right) \\
& +\sum_{q=1}^{n} 4 \alpha_{p q} L_{g} \rho_{p_{l}}^{-1} e^{\lambda\left(\theta-t_{0}\right)} \eta_{p q} e^{\lambda t}\left(\left|z_{q}^{R}(t)\right|+\left|z_{q}^{I}(t)\right|+\left|z_{q}^{J}(t)\right|\right.
\end{aligned}
$$


Li et al. Advances in Difference Equations

(2019) 2019:63

Page 16 of 23

$$
\begin{aligned}
& \left.+\left|z_{q}^{K}(t)\right|\right) d u+\sum_{q=1}^{n} 4 \beta_{p q} L_{g} \rho_{p l}^{-1} e^{\lambda\left(\theta-t_{0}\right)} \xi_{p q} e^{\lambda t}\left(\left|z_{q}^{R}(t)\right|+\left|z_{q}^{I}(t)\right|\right. \\
& \left.\left.+\left|z_{q}^{J}(t)\right|+\left|z_{q}^{K}(t)\right|\right) d u\right\} \\
& \leq \sum_{p=1}^{n}\left\{\left(\lambda-a_{p}^{-}\right) \rho_{p_{l}}^{-1} e^{\lambda\left(t-t_{0}\right)}\left|z_{p}^{l}(t)\right|+\sum_{q=1}^{n}\left(\frac{4 b_{p q} L_{f} e^{\lambda \theta}}{1-\dot{\tau}_{p q}}\right.\right. \\
& \left.+4 \alpha_{p q} L_{g} e^{\lambda \theta} \eta_{p q}+4 \beta_{p q} L_{g} e^{\lambda \theta} \xi_{p q}\right) \\
& \left.\quad \times \rho_{p_{l}}^{-1} e^{\lambda\left(t-t_{0}\right)}\left(\left|z_{q}^{R}(t)\right|+\left|z_{q}^{I}(t)\right|+\left|z_{q}^{J}(t)\right|+\left|z_{q}^{K}(t)\right|\right)\right\}, \quad l \in \Upsilon .
\end{aligned}
$$

From this and $\left(H_{5}\right)$, we have

$$
\begin{aligned}
D^{+} V( & t) \\
= & D^{+} V_{R}(t)+D^{+} V_{I}(t)+D^{+} V_{J}(t)+D^{+} V_{K}(t) \\
\leq & \sum_{p=1}^{n}\left\{\left(\lambda-a_{p}^{-}\right) e^{\lambda\left(t-t_{0}\right)}\left(\rho_{p_{R}}^{-1}\left|z_{p}^{R}(t)\right|+\rho_{p_{I}}^{-1}\left|z_{p}^{I}(t)\right|+\rho_{p_{J}}^{-1}\left|z_{p}^{J}(t)\right|+\rho_{p_{K}}^{-1}\left|z_{p}^{K}(t)\right|\right)\right. \\
& +\sum_{q=1}^{n}\left(\frac{4 b_{p q} L_{f} e^{\lambda \theta}}{1-\dot{\tau}_{p q}}+4 \alpha_{p q} L_{g} e^{\lambda \theta} \eta_{p q}+4 \beta_{p q} L_{g} e^{\lambda \theta} \xi_{p q}\right) e^{\lambda\left(t-t_{0}\right)}\left(\rho_{p_{R}}^{-1}+\rho_{p_{I}}^{-1}\right. \\
& \left.\left.+\rho_{p_{J}}^{-1}+\rho_{p_{K}}^{-1}\right)\left(\left|z_{q}^{R}(t)\right|+\left|z_{q}^{I}(t)\right|+\left|z_{q}^{J}(t)\right|+\left|z_{q}^{K}(t)\right|\right)\right\} \\
\leq & \sum_{p=1}^{n}\left\{\left(\lambda-a_{p}^{-}\right) \frac{1}{\rho^{-}} e^{\lambda\left(t-t_{0}\right)}\left(\left|z_{p}^{R}(t)\right|+\left|z_{p}^{I}(t)\right|+\left|z_{p}^{J}(t)\right|+\left|z_{p}^{K}(t)\right|\right)\right. \\
& +\sum_{q=1}^{n}\left(\frac{16 b_{q p} L_{f} e^{\lambda \theta}}{1-\dot{\tau}_{q p}}+16 \alpha_{q p} L_{g} e^{\lambda \theta} \eta_{q p}+16 \beta_{q p} L_{g} e^{\lambda \theta} \xi_{q p}\right) \frac{1}{\rho^{-}} e^{\lambda\left(t-t_{0}\right)}\left(\left|z_{p}^{R}(t)\right|\right. \\
\leq & 0 . \\
& \left.+\left|z_{p}^{I}(t)\right|+\left|z_{p}^{J}(t)\right|+\left|z_{p}^{K}(t)\right|\right\} \\
= & \sum_{p=1}^{n}\left\{\lambda-a_{p}^{-}+\sum_{q=1}^{n}\left(\frac{16 b_{q p} L_{f} e^{\lambda \theta}}{1-\dot{\tau}_{q p}}+16 \alpha_{q p} L_{g} e^{\lambda \theta} \eta_{q p}+16 \beta_{q p} L_{g} e^{\lambda \theta} \xi_{q p}\right)\right\} \\
& \\
& \\
& \\
&
\end{aligned}
$$

Hence, $V(t) \leq V\left(t_{0}\right)$ for $t \geq t_{0}$. By the expression of $V(t)$, we can obtain

$$
\begin{aligned}
V(t) & \geq \sum_{p=1}^{n} e^{\lambda\left(t-t_{0}\right)}\left(\rho_{p_{R}}^{-1}\left|z_{p}^{R}(t)\right|+\rho_{p_{I}}^{-1}\left|z_{p}^{I}(t)\right|+\rho_{p_{J}}^{-1}\left|z_{p}^{J}(t)\right|+\rho_{p_{K}}^{-1}\left|z_{p}^{K}(t)\right|\right) \\
& \geq 4 n e^{\lambda\left(t-t_{0}\right)}\|z(t)\|=4 n e^{\lambda\left(t-t_{0}\right)}\|x(t)-y(t)\|
\end{aligned}
$$


and

$$
\begin{aligned}
& V_{l}\left(t_{0}\right)=\sum_{p=1}^{n}\left\{\rho_{p_{l}}^{-1}\left|z_{p}^{l}\left(t_{0}\right)\right|+\sum_{q=1}^{n} \frac{4 b_{p q} L_{f} \rho_{p_{l}}^{-1} e^{\lambda\left(\theta-t_{0}\right)}}{1-\dot{\tau}_{p q}}\right. \\
& \times \int_{t_{0}-\tau_{p q}\left(t_{0}\right)}^{t_{0}} e^{\lambda s}\left(\left|z_{q}^{R}(s)\right|+\left|z_{q}^{I}(s)\right|+\left|z_{q}^{J}(s)\right|+\left|z_{q}^{K}(s)\right|\right) d s \\
& +\sum_{q=1}^{n} 4 \alpha_{p q} L_{g} \rho_{p_{l}}^{-1} e^{\lambda\left(\theta-t_{0}\right)} \int_{-\eta_{p q}}^{0} \int_{t_{0}+u}^{t_{0}} e^{\lambda s}\left(\left|z_{q}^{R}(s)\right|+\left|z_{q}^{I}(s)\right|+\left|z_{q}^{J}(s)\right|\right. \\
& \left.+\left|z_{q}^{K}(s)\right|\right) d s d u+\sum_{q=1}^{n} 4 \beta_{p q} L_{g} \rho_{p_{l}}^{-1} e^{\lambda\left(\theta-t_{0}\right)} \int_{-\xi_{p q}}^{0} \int_{t_{0}+u}^{t_{0}} e^{\lambda s}\left(\left|z_{q}^{R}(s)\right|\right. \\
& \left.\left.+\left|z_{q}^{I}(s)\right|+\left|z_{q}^{J}(s)\right|+\left|z_{q}^{K}(s)\right|\right) d s d u\right\} \\
& =\sum_{p=1}^{n}\left\{\rho_{p_{l}}^{-1}\left|z_{p}^{l}\left(t_{0}\right)\right|+\sum_{q=1}^{n} \frac{4 b_{p q} L_{f} \rho_{p_{R}} \rho_{p_{l}}^{-1} e^{\lambda\left(\theta-t_{0}\right)}}{1-\dot{\tau}_{p q}}\right. \\
& \times \int_{t_{0}-\tau_{p q}\left(t_{0}\right)}^{t_{0}} e^{\lambda s} \rho_{p_{R}}^{-1}\left|z_{q}^{R}(s)\right| d s \\
& +\sum_{q=1}^{n} \frac{4 b_{p q} L_{f} \rho_{p_{I}} \rho_{p_{l}}^{-1} e^{\lambda\left(\theta-t_{0}\right)}}{1-\dot{\tau}_{p q}} \int_{t_{0}-\tau_{p q}\left(t_{0}\right)}^{t_{0}} e^{\lambda s} \rho_{p_{I}}^{-1}\left|z_{q}^{I}(s)\right| d s \\
& +\sum_{q=1}^{n} \frac{4 b_{p q} L_{f} \rho_{p_{J}} \rho_{p_{l}}^{-1} e^{\lambda\left(\theta-t_{0}\right)}}{1-\dot{\tau}_{p q}} \int_{t_{0}-\tau_{p q}\left(t_{0}\right)}^{t_{0}} e^{\lambda s} \rho_{p_{J}}^{-1}\left|z_{q}^{J}(s)\right| d s \\
& +\sum_{q=1}^{n} \frac{4 b_{p q} L_{f} \rho_{p_{K}} \rho_{p_{l}}^{-1} e^{\lambda\left(\theta-t_{0}\right)}}{1-\dot{\tau}_{p q}} \int_{t_{0}-\tau_{p q}\left(t_{0}\right)}^{t_{0}} e^{\lambda s} \rho_{p_{K}}^{-1}\left|z_{q}^{K}(s)\right| d s \\
& +\sum_{q=1}^{n} 4 \alpha_{p q} L_{g} \rho_{p_{R}} \rho_{p_{l}}^{-1} e^{\lambda\left(\theta-t_{0}\right)} \int_{-\eta_{p q}}^{0} \int_{t_{0}+u}^{t_{0}} e^{\lambda s} \rho_{p_{R}}^{-1}\left|z_{q}^{R}(s)\right| d s d u \\
& +\sum_{q=1}^{n} 4 \alpha_{p q} L_{g} \rho_{p_{I}} \rho_{p_{l}}^{-1} e^{\lambda\left(\theta-t_{0}\right)} \int_{-\eta_{p q}}^{0} \int_{t_{0}+u}^{t_{0}} e^{\lambda s} \rho_{p_{I}}^{-1}\left|z_{q}^{I}(s)\right| d s d u \\
& +\sum_{q=1}^{n} 4 \alpha_{p q} L_{g} \rho_{p_{J}} \rho_{p_{l}}^{-1} e^{\lambda\left(\theta-t_{0}\right)} \int_{-\eta_{p q}}^{0} \int_{t_{0}+u}^{t_{0}} e^{\lambda s} \rho_{p_{J}}^{-1}\left|z_{q}^{J}(s)\right| d s d u \\
& +\sum_{q=1}^{n} 4 \alpha_{p q} L_{g} \rho_{p_{K}} \rho_{p_{l}}^{-1} e^{\lambda\left(\theta-t_{0}\right)} \int_{-\eta_{p q}}^{0} \int_{t_{0}+u}^{t_{0}} e^{\lambda s} \rho_{p_{K}}^{-1}\left|z_{q}^{K}(s)\right| d s d u \\
& +\sum_{q=1}^{n} 4 \beta_{p q} L_{g} \rho_{p_{R}} \rho_{p_{l}}^{-1} e^{\lambda\left(\theta-t_{0}\right)} \int_{-\xi_{p q}}^{0} \int_{t_{0}+u}^{t_{0}} e^{\lambda s} \rho_{p_{R}}^{-1}\left|z_{q}^{R}(s)\right| d s d u \\
& +\sum_{q=1}^{n} 4 \beta_{p q} L_{g} \rho_{p_{I}} \rho_{p_{l}}^{-1} e^{\lambda\left(\theta-t_{0}\right)} \int_{-\xi_{p q}}^{0} \int_{t_{0}+u}^{t_{0}} e^{\lambda s} \rho_{p_{I}}^{-1}\left|z_{q}^{I}(s)\right| d s d u \\
& +\sum_{q=1}^{n} 4 \beta_{p q} L_{g} \rho_{p_{J}} \rho_{p_{l}}^{-1} e^{\lambda\left(\theta-t_{0}\right)} \int_{-\xi_{p q}}^{0} \int_{t_{0}+u}^{t_{0}} e^{\lambda s} \rho_{p_{J}}^{-1}\left|z_{q}^{J}(s)\right| d s d u
\end{aligned}
$$




$$
\begin{aligned}
& \left.+\sum_{q=1}^{n} 4 \beta_{p q} L_{g} \rho_{p_{K}} \rho_{p_{l}}^{-1} e^{\lambda\left(\theta-t_{0}\right)} \int_{-\xi_{p q}}^{0} \int_{t_{0}+u}^{t_{0}} e^{\lambda s} \rho_{p_{K}}^{-1}\left|z_{q}^{K}(s)\right| d s d u\right\} \\
\leq & \sum_{p=1}^{n}\left\{1+\sum_{q=1}^{n}\left(\frac{16 \tau_{p q} b_{p q} L_{f} \rho_{p_{l}}^{-1}}{1-\dot{\tau}_{p q}}+8 \eta_{p q}^{2} \alpha_{p q} L_{g} \rho_{p_{l}}^{-1}\right.\right. \\
& \left.\left.+8 \xi_{p q}^{2} \beta_{p q} L_{g} \rho_{p_{l}}^{-1}\right) \rho^{+} e^{\lambda \theta}\right\}\|\varphi-\psi\| .
\end{aligned}
$$

Denote

$$
\begin{aligned}
M= & \max _{\substack{1 \leq p \leq n \\
l \in \Upsilon}}\left\{1+\sum_{q=1}^{n}\left(\frac{16 \tau_{p q} b_{p q} L_{f} \rho_{p_{l}}^{-1}}{1-\dot{\tau}_{p q}}+8 \eta_{p q}^{2} \alpha_{p q} L_{g} \rho_{p_{l}}^{-1}\right.\right. \\
& \left.\left.+8 \xi_{p q}^{2} \beta_{p q} L_{g} \rho_{p_{l}}^{-1}\right) \rho^{+} e^{\lambda \theta}\right\}>0 .
\end{aligned}
$$

Thus, we have

$$
4 n e^{\lambda\left(t-t_{0}\right)}\|x(t)-y(t)\| \leq V\left(t_{0}\right) \leq V(t) \leq 4 n M\|\varphi-\psi\|, \quad t \geq t_{0},
$$

that is,

$$
\|x(t)-y(t)\| \leq M\|\varphi-\psi\| e^{-\lambda\left(t-t_{0}\right)}, \quad t \geq t_{0}
$$

Therefore, the $\omega$-periodic solution $x(t)$ of system (2) is globally exponentially stable. In view of Remark 1 , we see that the $\omega$-periodic solution of system (1) is also globally exponentially stable. The uniqueness of the $\omega$-periodic solution follows from the global exponential stability. The proof is complete.

\section{An illustrative example}

In this section, an example is given to illustrate the effectiveness of our results obtained in this paper.

Example 1 Consider the following QVFCNN:

$$
\begin{aligned}
\dot{x}_{p}(t)= & -a_{p}(t) x_{p}(t)+\sum_{q=1}^{n} b_{p q}(t) f_{q}\left(x_{q}\left(t-\tau_{p q}(t)\right)\right)+\sum_{q=1}^{n} d_{p q}(t) \mu_{q}(t) \\
& +\bigwedge_{q=1}^{n} \alpha_{p q}(t) \int_{t-\eta_{p q}(t)}^{t} g_{q}(s, x) d s+\bigvee_{q=1}^{n} \beta_{p q}(t) \int_{t-\xi p q}^{t} g_{q}(s, x) d s \\
& +\bigwedge_{q=1}^{n} T_{p q}(t) \mu_{q}(t)+\bigvee_{q=1}^{n} S_{p q}(t) \mu_{q}(t)+I_{p}(t),
\end{aligned}
$$

where

$$
\dot{x}_{p}(t)=\dot{x}_{p}^{R}(t)+i \dot{x}_{p}^{I}(t)+j \dot{x}_{p}^{I}(t)+k \dot{x}_{p}^{K}(t) \in \mathbb{Q}, \quad p=1,2,
$$




$$
\begin{aligned}
& f_{q}\left(x_{q}\right)=\frac{1}{4} \sin \left(x_{q}^{R}+\frac{1}{2} x_{q}^{I}\right)+\frac{1}{2} j \sin \left(x_{q}^{R}+x_{q}^{I}+x_{q}^{J}+x_{q}^{K}\right)+\frac{1}{4} k x_{q}^{J}, \\
& g_{q}\left(x_{q}\right)=\frac{1}{4} j\left(x_{q}^{R}+x_{q}^{I}+x_{q}^{J}+x_{q}^{K}\right)+\frac{1}{4} k \sin \left(x_{q}^{R}+x_{q}^{K}\right), \\
& {\left[\begin{array}{l}
a_{1}(t) \\
a_{2}(t)
\end{array}\right]=\left[\begin{array}{c}
\sin ^{2}(20 t)+20 \\
-5 \cos ^{2}(20 t)+25
\end{array}\right]} \\
& {\left[\begin{array}{l}
I_{1}(t) \\
I_{2}(t)
\end{array}\right]=\left[\begin{array}{c}
\sin 40 t+i \sin 40 t+j \cos ^{3}(40 t)+\frac{1}{3} k \cos 40 t \\
\cos 40 t+i \sin 40 t+j \cos 40 t+k \sin ^{5}(40 t)
\end{array}\right],} \\
& {\left[\begin{array}{ll}
b_{11}(t) & b_{12}(t) \\
b_{21}(t) & b_{22}(t)
\end{array}\right]} \\
& =\frac{1}{4}\left[\begin{array}{cc}
\cos 40 t+i \sin 80 t+j \sin ^{2}(20 t) & \sin 40 t+k \cos 40 t \\
i \cos 40 t+j \sin 80 t-k \cos ^{3}(40 t) & \cos ^{2}(20 t)+j \cos ^{4}(20 t)+k \sin 40 t
\end{array}\right], \\
& {\left[\begin{array}{ll}
d_{11}(t) & d_{12}(t) \\
d_{21}(t) & d_{22}(t)
\end{array}\right]} \\
& =\left[\begin{array}{cc}
\sin 40 t+\frac{1}{2} i \cos 40 t+k \sin ^{2}(20 t) & \frac{2}{3} \sin 40 t+j \cos ^{3}(40 t)-k \cos 40 t \\
\cos 40 t+j \cos ^{3}(40 t)+\frac{1}{2} k \sin 40 t & \sin ^{3}(40 t)-j \cos 40 t+k \sin 40 t
\end{array}\right], \\
& {\left[\begin{array}{ll}
\alpha_{11}(t) & \alpha_{12}(t) \\
\alpha_{21}(t) & \alpha_{22}(t)
\end{array}\right]} \\
& =\frac{1}{4}\left[\begin{array}{cc}
\cos ^{5}(20 t)+i \sin 40 t+k \cos 40 t & i \sin 40 t \\
i \sin 40 t+\frac{1}{2} k \cos ^{2}(20 t) & \sin 40 t+i \cos 40 t+j \sin ^{3}(40 t)
\end{array}\right], \\
& {\left[\begin{array}{ll}
\beta_{11}(t) & \beta_{12}(t) \\
\beta_{21}(t) & \beta_{22}(t)
\end{array}\right]} \\
& =\frac{1}{4}\left[\begin{array}{cc}
\cos ^{5}(40 t)+\frac{1}{2} i \sin 40 t+k \cos 40 t & i \sin ^{2}(20 t) \\
\frac{1}{\sqrt{2}} i \sin 40 t+k \cos 40 t & \sin 40 t+i \cos 40 t+j \sin ^{3}(40 t)
\end{array}\right], \\
& {\left[\begin{array}{ll}
T_{11}(t) & T_{12}(t) \\
T_{21}(t) & T_{22}(t)
\end{array}\right]=\left[\begin{array}{cc}
\frac{1}{3} \cos ^{2}(20 t)+i \cos 40 t+k \frac{1}{3} & \frac{1}{2} \sin ^{2}(20 t)+j \sin ^{3}(40 t) \\
\frac{1}{2} \sin 40 t+\frac{1}{4} k \cos 40 t & \frac{1}{4}+\frac{1}{4} k \sin 40 t
\end{array}\right],} \\
& {\left[\begin{array}{ll}
S_{11}(t) & S_{12}(t) \\
S_{21}(t) & S_{22}(t)
\end{array}\right]=\left[\begin{array}{cc}
\frac{1}{5} \cos 40 t+k \sin ^{2}(20 t) & \frac{1}{2} \sin ^{2}(20 t)+j \cos ^{2}(20 t) \\
\frac{1}{2} \sin ^{4}(20 t)+i \cos ^{2}(20 t) & \frac{1}{4}+k \sin 40 t
\end{array}\right],} \\
& {\left[\begin{array}{l}
\mu_{1}(t) \\
\mu_{2}(t)
\end{array}\right]=\left[\begin{array}{c}
\frac{1}{3} \cos 40 t+i \sin 40 t+k \cos 40 t \\
\frac{1}{2} \sin 40 t+k \cos 40 t
\end{array}\right] \text {, }} \\
& {\left[\begin{array}{ll}
\tau_{11}(t) & \tau_{12}(t) \\
\tau_{21}(t) & \tau_{22}(t)
\end{array}\right]=\left[\begin{array}{cc}
\frac{1}{40} \cos ^{2}(20 t) & \frac{1}{40} \sin ^{2}(20 t) \\
\frac{1}{40} \sin ^{2}(20 t) & \frac{1}{4}
\end{array}\right],} \\
& {\left[\begin{array}{ll}
\eta_{11}(t) & \eta_{12}(t) \\
\eta_{21}(t) & \eta_{22}(t)
\end{array}\right]=\left[\begin{array}{ll}
\frac{1}{2} \sin ^{2}(20 t) & \frac{1}{4} \cos ^{2}(20 t) \\
\frac{1}{4} \sin ^{2}(20 t) & \frac{1}{4} \sin ^{2}(20 t)
\end{array}\right],} \\
& {\left[\begin{array}{ll}
\xi_{11}(t) & \xi_{12}(t) \\
\xi_{21}(t) & \xi_{22}(t)
\end{array}\right]=\left[\begin{array}{cc}
\frac{1}{4} & \frac{1}{3} \cos ^{2}(20 t) \\
\frac{1}{2} \cos ^{2}(20 t) & \frac{1}{2} \sin ^{2}(20 t)
\end{array}\right] \text {. }}
\end{aligned}
$$



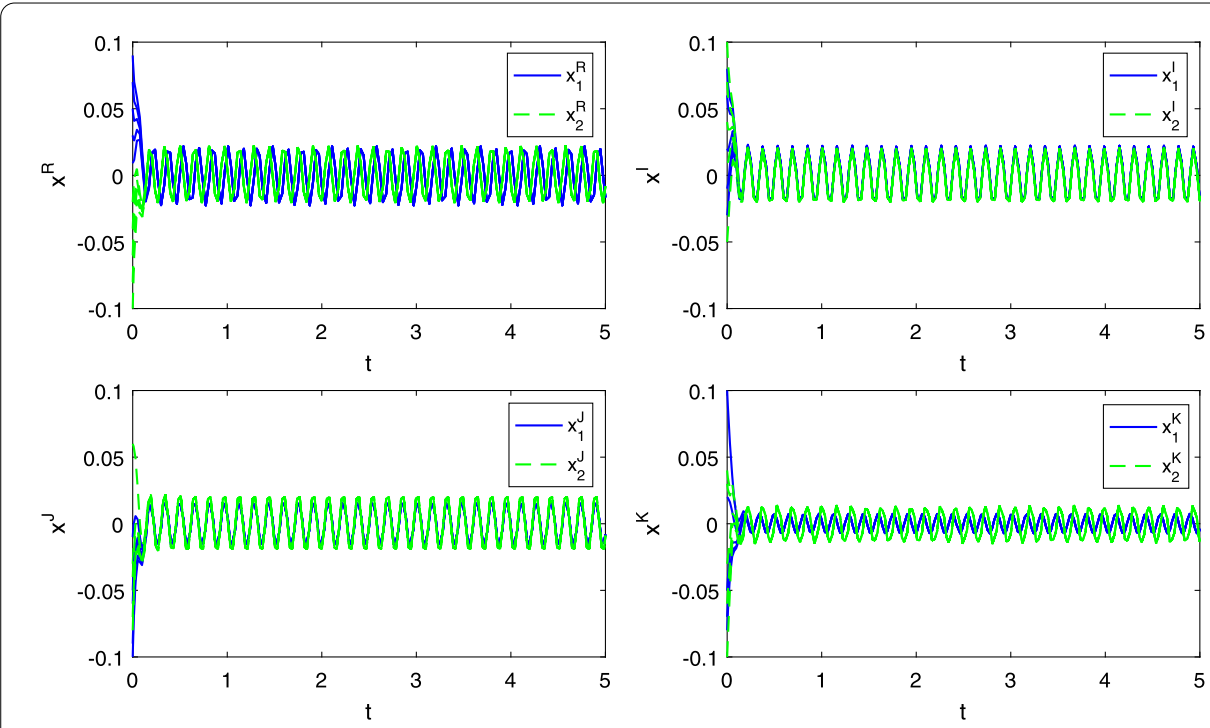

Figure 1 The states of four parts of $x_{1}$ and $x_{2}$

By computing, we have

$$
\begin{aligned}
& a_{1}^{-}=a_{2}^{-}=20, \quad L_{f}=\frac{1}{8}, \quad L_{g}=\frac{1}{4}, \quad \theta=\frac{1}{2}, \quad \omega=\frac{\pi}{20}, \\
& {\left[\begin{array}{ll}
b_{11} & b_{12} \\
b_{21} & b_{22}
\end{array}\right]=\left[\begin{array}{ll}
\alpha_{11} & \alpha_{12} \\
\alpha_{21} & \alpha_{22}
\end{array}\right]=\left[\begin{array}{ll}
\beta_{11} & \beta_{12} \\
\beta_{21} & \beta_{22}
\end{array}\right]=\left[\begin{array}{cc}
\frac{1}{4} & \frac{1}{4} \\
\frac{1}{4} & \frac{1}{4}
\end{array}\right],} \\
& {\left[\begin{array}{ll}
\dot{\tau}_{11}(t) & \dot{\tau}_{12}(t) \\
\dot{\tau}_{21}(t) & \dot{\tau}_{22}(t)
\end{array}\right]=\left[\begin{array}{cc}
-\frac{1}{2} \sin 40 t & \frac{1}{2} \sin 40 t \\
\frac{1}{2} \sin 40 t & 0
\end{array}\right],} \\
& {\left[\begin{array}{ll}
\tau_{11} & \tau_{12} \\
\tau_{21} & \tau_{22}
\end{array}\right]=\left[\begin{array}{cc}
\frac{1}{40} & \frac{1}{40} \\
\frac{1}{40} & \frac{1}{4}
\end{array}\right], \quad\left[\begin{array}{cc}
\eta_{11} & \eta_{12} \\
\eta_{21} & \eta_{22}
\end{array}\right]=\left[\begin{array}{cc}
\frac{1}{2} & \frac{1}{4} \\
\frac{1}{4} & \frac{1}{4}
\end{array}\right],} \\
& {\left[\begin{array}{ll}
\xi_{11} & \xi_{12} \\
\xi_{21} & \xi_{22}
\end{array}\right]=\left[\begin{array}{ll}
\frac{1}{4} & \frac{1}{3} \\
\frac{1}{2} & \frac{1}{2}
\end{array}\right], \quad\left[\begin{array}{ll}
\dot{\tau}_{11} & \dot{\tau}_{12} \\
\dot{\tau}_{21} & \dot{\tau}_{22}
\end{array}\right]=\left[\begin{array}{cc}
\frac{1}{2} & \frac{1}{2} \\
\frac{1}{2} & 0
\end{array}\right] .}
\end{aligned}
$$

Take $\lambda=1$, then we have

$$
\max _{1 \leq p \leq 2}\left\{\frac{4 \omega}{1-e^{-\omega \bar{a}_{p}}}\left[\sum_{q=1}^{n}\left(b_{p q} L_{f}+\alpha_{p q} L_{g} \theta+\beta_{p q} L_{g} \theta\right)\right]\right\}=0.246<\frac{1}{4}
$$

and

$$
\max _{1 \leq p \leq 2}\left\{\lambda-a_{p}^{-}+\sum_{q=1}^{n} 16 e^{\lambda \theta}\left(\frac{b_{q p} L_{f}}{1-\dot{\tau}_{q p}}+\left(\alpha_{q p} \eta_{q p}+\beta_{q p} \xi_{q p}\right) L_{g}\right)\right\}=-17.28 \leq 0 .
$$

Therefore, all the conditions of Theorem 2 are satisfied. According to Theorem 2, system (8) has a unique $\frac{\pi}{20}$-periodic solution and it is globally exponentially stable (see Figs. 1-3). 

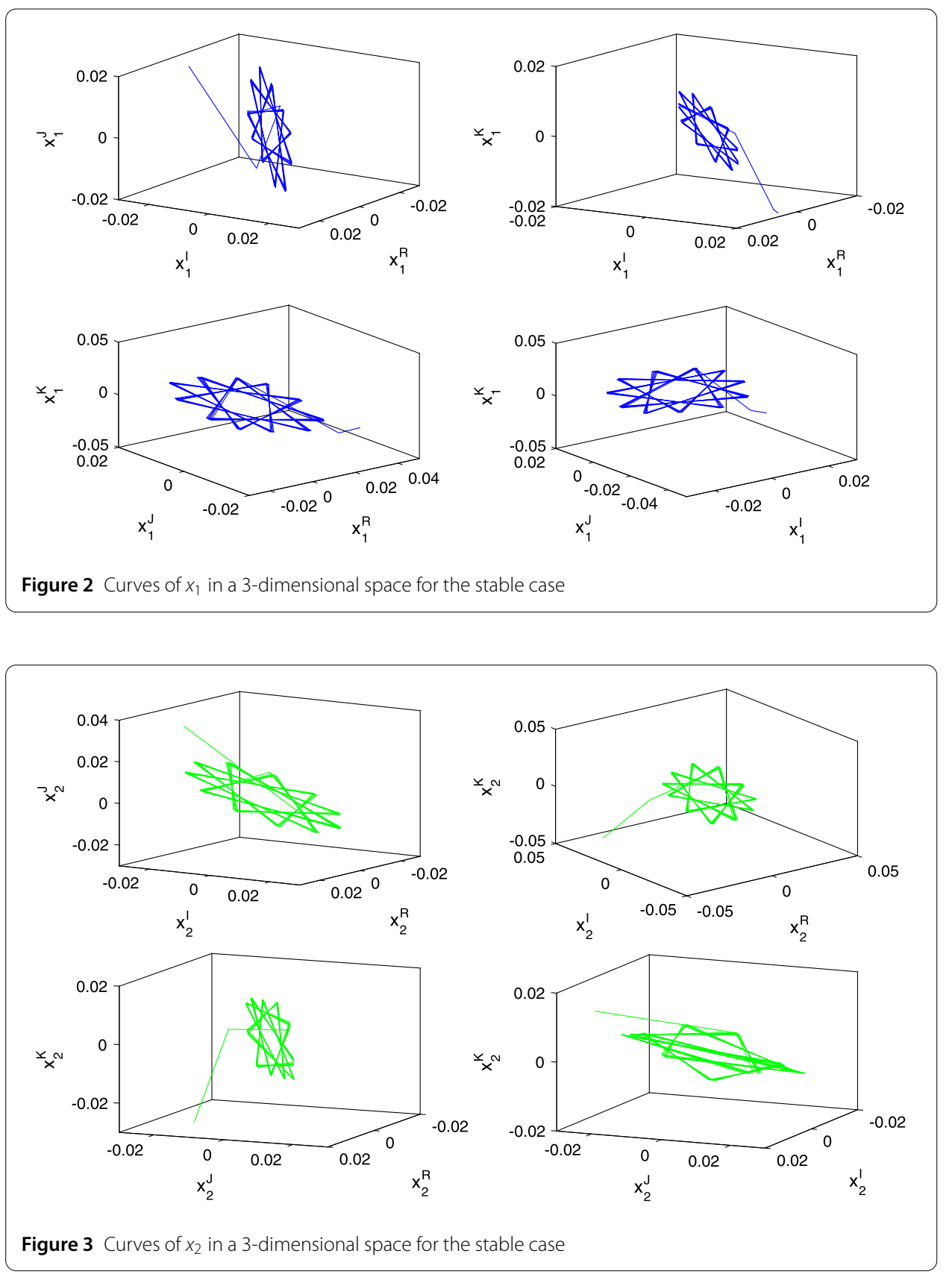

Remark 2 Even when QVFCNN (8) degenerates into a real-valued system, papers [23, $32,45-47$ ] cannot be used to judge that (8) has a globally exponentially stable periodic solution.

\section{Conclusions}

In this paper, by using the Schauder fixed point theorem and constructing a suitable Lyapunov function, we investigated the existence and global exponential stability of periodic solutions for QVFCNNs with time-varying delays. To the best of our knowledge, this is the first paper to study the periodic solutions of QVFCNNs. Our results are new and supplement some previously known ones even when system (1) degenerates into a real-valued 
system. Our methods used in this paper can be used to study other types of quaternionvalued neural networks such as Hopfield neural networks, BAM neural networks, CohenGrossberg neural networks, and so on.

\section{Acknowledgements}

Not applicable.

\section{Funding}

This work is supported by the National Natural Sciences Foundation of People's Republic of China under Grant 11861072.

\section{Availability of data and materials}

Data sharing not applicable to this article as no datasets were generated or analysed during the current study.

\section{Ethics approval and consent to participate}

Not applicable.

\section{Competing interests}

The authors declare that they have no competing interests.

\section{Consent for publication}

Not applicable.

\section{Authors' contributions}

The three authors contributed equally to the manuscript and typed, read, and approved the final manuscript.

\section{Author details}

'Department of Mathematics, Yunnan University, Yunnan, China. ${ }^{2}$ School of Mathematics and Computer Science, Yunnan Nationalities University, Yunnan, China.

\section{Publisher's Note}

Springer Nature remains neutral with regard to jurisdictional claims in published maps and institutional affiliations.

Received: 12 December 2018 Accepted: 6 February 2019 Published online: 15 February 2019

\section{References}

1. Adler, S.: Quaternionic Quantum Mechanics and Quantum Fields. Oxford University Press, New York (1995)

2. Adler, S.: Quaternionic quantum field theory. Commun. Math. Phys. 104(4), 611-656 (1986)

3. Leo, S., Ducati, G.: Delay time in quaternionic quantum mechanics. J. Math. Phys. 53(2), 022102 (2012)

4. Udwadia, F., Schttle, A.: An alternative derivation of the quaternion equations of motion for rigid-body rotational dynamics. J. Appl. Mech. 77(4), 044505 (2010)

5. Gibbon, J.D., Holm, D.D., Kerr, R.M.: Quaternions and particle dynamics in the Euler fluid equations. Nonlinearity 19 , 1969-1983 (2006)

6. Handson, A., Ma, H.: Quaternion frame approach to stream line visualization. IEEE Trans. Vis. Comput. Graph. 1(2), 164-172 (1995)

7. Ell, T., Sangwine, S.J.: Hypercomplex Fourier transforms of color images. IEEE Trans. Image Process. 16(1), 22-35 (2007)

8. Miron, S., Bihan, N.L., Mars, J.I.: Quaternion-music for vector-sensor array processing. IEEE Trans. Signal Process. 54(4), 1218-1229 (2006)

9. Took, C.C., Strbac, G., Aihara, K., Mandic, D.: Quaternion-valued short-term joint forecasting of three-dimensional wind and atmospheric parameters. Renew. Energy 36(6), 1754-1760 (2011)

10. Isokawa, T., Kusakabe, T., Matsui, N., Peper, F.: Quaternion neural network and its application. In: Knowledge-Based Intelligent Information and Engineering Systems, pp. 318-324. Springer, Berlin (2003)

11. Shu, H., Song, Q., Liu, Y., Zhao, Z., Alsaadi, F.E.: Global $\mu$-stability of quaternion-valued neural networks with non-differentiable time-varying delays. Neurocomputing 247, 202-212 (2017)

12. Song, Q., Chen, X.: Multistability analysis of quaternion-valued neural networks with time delays. IEEE Trans. Neural Netw. Learn. Syst. 29(11), 5430-5440 (2018)

13. Liu, Y., Zhang, D., Lu, J.: Global exponential stability for quaternion-valued recurrent neural networks with time-varying delays. Nonlinear Dyn. 87, 553-565 (2017)

14. Isokawa, T., Matsui, N., Nishimura, H.: Quaternionic neural networks: fundamental properties and applications. In: Complex Valued Neural Networks: Util. High Dimens. Parameters (2009) https://doi.org/10.4018/978-1-60566-214-5.ch016

15. Matsui, N., Isokawa, T., Kusamichi, H., Peper, F., Nishimura, H.: Quaternion neural network with geometrical operators J. Intell. Fuzzy Syst. 15(3-4), 149-164 (2004)

16. Isokawa, T., Kusakabe, T., Matsui, N., Peper, F.: Quaternion neural network and its application. In: Knowledge-Based Intelligent Information and Engineering Systems. vol. 2774, pp. 318-324. Springer, Berlin (2003)

17. Grossberg, S.: Neural Networks and Natural Intelligence. MIT Press, Boston (1992)

18. Borkar, V.S., Soumyanatha, K.: An analog scheme for fixed point computation-Part I: theory. IEEE Trans. Circuits Syst. I 44, 351-355 (1997)

19. Schmidhuber, J.: Deep learning in neural networks: an overview. Neural Netw. 61, 85-117 (2015) 
20. Pakdaman, M., Ahmadian, A., Effati, S., Salahshour, S., Baleanu, D.: Solving differential equations of fractional order using an optimization technique based on training artificial neural network. Appl. Math. Comput. 293, 81-95 (2017)

21. Liu, Y., Zhang, D., Lu, J., Cao, J.: Global $\mu$-stability criteria for quaternion-valued neural networks with unbounded time-varying delays. Inf. Sci. 360, 273-288 (2016)

22. Liu, Y., Zhang, D., Lu, J.: Global exponential stability for quaternion-valued recurrent neural networks with time-varying delays. Nonlinear Dyn. 87, 553-565 (2017)

23. Li, Y., Qin, J.: Existence and global exponential stability of periodic solutions for quaternion-valued cellular neural networks with time-varying delays. Neurocomputing 292, 91-103 (2018)

24. Li, Y., Meng, X.: Existence and global exponential stability of pseudo almost periodic solutions for neutral type quaternion-valued neural networks with delays in the leakage term on time scales. Complexity 2017, Article ID 9878369 (2017)

25. Yang, T., Yang, L., Wu, C.W., Chua, L.O.: Fuzzy cellular neural networks: theory. In: Proceedings of the IEEE International Workshop on Cellular Neural Networks and Applications, pp. 181-186 (1996)

26. Yang, T., Yang, L., Wu, C.W., Chua, L.O.: Fuzzy cellular neural networks: applications. In: Proceedings of the IEEE International Work Shop on Cellular Neural Networks and Applications, pp. 225-230 (1996)

27. Ahmadian, A., Salahshour, S., Chan, C.S.: Fractional differential systems: a fuzzy solution based on operational matrix of shifted Chebyshev polynomials and its applications. IEEE Trans. Fuzzy Syst. 25(1), 218-236 (2017)

28. Ahmadian, A., Salahshour, S., Chan, C.S., Baleanu, D.: Numerical solutions of fuzzy differential equations by an efficient Runge-Kutta method with generalized differentiability. Fuzzy Sets Syst. 331, 47-67 (2018)

29. Salahshour, S., Ahmadian, A., Senu, N., Baleanu, D., Agarwal, P.: On analytical solutions of the fractional differential equation with uncertainty: application to the Basset problem. Entropy 17(2), 885-902 (2015)

30. Li, Y., Zhang, T.: Global exponential stability of fuzzy interval delayed neural networks with impulses on time scales. Int. J. Neural Syst. 19(06), 449-456 (2009)

31. Yang, T., Yang, L.: The global stability of fuzzy cellular neural networks. IEEE Trans. Circuits Syst. 43(10), 880-883 (1996)

32. Yuan, K., Cao, J., Deng, J.: Exponential stability and periodic solutions of fuzzy cellular neural networks with time-varying delays. Neurocomputing 69(13), 1619-1627 (2006)

33. Zhang, Q., Xiang, R.: Global asymptotic stability of fuzzy cellular neural networks with time-varying delays. Phys. Lett. A 372(22), 3971-3977 (2008)

34. Zhou, L., Hu, G.: Global exponential periodicity and stability of cellular neural networks with variable and distributed delays. Appl. Math. Comput. 195(2), 402-411 (2008)

35. Liu, Z., Zhang, H., Wang, Z.: Novel stability criterions of a new fuzzy cellular neural networks with time-varying delays. Neurocomputing 72(4-6), 1056-1064 (2009)

36. Bao, H.: Existence and exponential stability of periodic solution for BAM fuzzy Cohen-Grossberg neural networks with mixed delays. Neural Process. Lett. 43(3), 871-885 (2016)

37. Song, Q., Wang, Z: Dynamical behaviors of fuzzy reaction-diffusion periodic cellular neural networks with variable coefficients and delays. Appl. Math. Model. 33(9), 3533-3545 (2009)

38. Rakkiyappan, R., Balasubramaniam, P.: On exponential stability results for fuzzy impulsive neural networks. Fuzzy Sets Syst. 161(13), 1823-1835 (2010)

39. Li, Y., Wang, C.: Existence and global exponential stability of equilibrium for discrete-time fuzzy BAM neural networks with variable delays and impulses. Fuzzy Sets Syst. 217, 62-79 (2013)

40. Abdurahman, A., Jiang, H., Teng, Z:: Finite-time synchronization for fuzzy cellular neural networks with time-varying delays. Fuzzy Sets Syst. 297, 96-111 (2016)

41. Jia, R.: Finite-time stability of a class of fuzzy cellular neural networks with multi-proportional delays. Fuzzy Sets Syst. 319, 70-80 (2017)

42. Wang, W.: Finite-time synchronization for a class of fuzzy cellular neural networks with time-varying coefficients and proportional delays. Fuzzy Sets Syst. 338, 40-49 (2018)

43. Huang, Z.D.: Almost periodic solutions for fuzzy cellular neural networks with time-varying delays. Neural Comput. Appl. 28, 2313-2320 (2017)

44. Huang, Z.D.: Almost periodic solutions for fuzzy cellular neural networks with multi-proportional delays. Int. J. Mach. Learn. Cybern. 8(4), 1323-1331 (2017)

45. Niu, S., Jiang, H., Teng, Z.: Exponential stability and periodic solutions of FCNNs with variable coefficients and time-varying delays. Neurocomputing 71(13-15), 2929-2936 (2008)

46. Niu, S., Jiang, H., Teng, Z.: Periodic oscillation of FCNNs with distributed delays and variable coefficients. Nonlinear Anal., Real World Appl. 10(3), 1540-1554 (2009)

47. Xu, C., Zhang, Q., Wu, Y.: Existence and exponential stability of periodic solution to fuzzy cellular neural networks with distributed delays. Int. J. Fuzzy Syst. 18(1), 41-51 (2016)

48. Amster, P.: Topological Methods in the Study of Boundary Value Problems. Springer, New York (2013) 